\title{
ANÁLISIS ECONÓMICO DE ALGUNAS ESTRATEGIAS DE ADAPTACIÓN AL CAMBIO CLIMÁTICO EN DRENAJE URBANO
}

\author{
María Eugenia Garat ${ }^{(1)}$, Adolfo Villanueva ${ }^{(2)}$ y Gerardo Riccardi ${ }^{(3)}$ \\ ${ }^{(1)}$ Universidad Tecnológica Nacional, Regional Concordia. Salta 277. Concordia, Entre Ríos, Argentina \\ ${ }^{(2)}$ Instituto Nacional del Agua, Centro Regional Litoral. Patricio Cullen 6161. Santa Fe, Argentina \\ ${ }^{(3)}$ Facultad de Ciencias Exactas, Ingeniería y Agrimensura. Universidad Nacional de Rosario. \\ Riobamba 250 bis. (2000). Rosario. Santa Fe. Argentina \\ email: aonvilla@gmail.com, gariccardi@gmail.com, eugarat@yahoo.com.ar
}

\begin{abstract}
RESUMEN
La gestión de los recursos hídricos debe trabajar sobre los problemas para la situación actual y desarrollar herramientas de planeamiento para escenarios de cambio climático, que incorporen el aumento de incertezas que el mismo genera. Como posibles formas de lidiar con los potenciales impactos del cambio climático se plantean cuatro estrategias de planeamiento de los sistemas de drenaje urbano: ignorar el impacto futuro, proyecto basado en los máximos incrementos previstos, adaptación por etapas, e incorporación de medidas de control en la fuente a lo largo del tiempo. Se simuló el funcionamiento de un sistema urbano para tres escenarios hipotéticos de impacto del cambio climático. Fueron cuantificados los costos de implementación de cada estrategia y los daños directos atribuibles a pérdidas por inundación. A partir de eso se estimaron los resultados económicos de cada estrategia y se realizó un análisis comparativo de las ventajas y desventajas de adoptar cada una de ellas. Un aspecto interesante del trabajo es que los resultados también son, al menos parcialmente, aplicables a las incertezas generales relacionadas a la lluvia, que van más allá de las originadas por el cambio climático, y ya se encuentran en condiciones de afectar los proyectos. Por ejemplo la utilización de series de lluvias muy cortas y poco representativas, o la variabilidad climática.
\end{abstract}

Palabras clave: Cambio climático, drenaje urbano, impacto hidráulico, impacto económico.

\begin{abstract}
Water resources management must work on the problems for the current situation and develop planning tools for scenarios of climate change, incorporating the increase of uncertainty that it generates. Possible ways to deal with the potential impacts of climate change are four strategies for planning urban drainage systems: ignoring the future impact, project based on the maximum expected increases, project adaptation by stages, and incorporation of source control measures. The operation of an urban system was simulated for three hypothetical climate change impact scenarios. The costs of implementing each strategy and the direct damages due to flood losses were quantified. From that, the economic results of each strategy were estimated and a comparative analysis was made of the advantages and disadvantages of adopting each one. An interesting aspect of this work is that the results are also at least partially applicable to general uncertainties related to rainfall, which go beyond those caused by climate change, and are already in a position to affect projects; for example, the use of very short and unrepresentative rainfall series or climatic variability.
\end{abstract}

Keywords: Climate change, urban drainage, hydraulic impact, economic impact. 


\section{INTRODUCCIÓN}

Los cambios observados en las tendencias climáticas en nuestro país reflejan un aumento de la precipitación media anual en la mayor parte del territorio argentino y el incremento de la frecuencia de precipitaciones extremas en las zonas centro y este del país (Barros et al., 2014). Sin embargo la magnitud de estos cambios y su impacto sobre las obras de infraestructura son inciertos, por lo cual no es posible asignar probabilidades cuantitativas a impactos específicos. Es decir que no existe seguridad sobre la magnitud del cambio, o el momento en que el mismo será alcanzado. Esto caracteriza la situación denominada "incerteza profunda" o "incerteza severa" (deep uncertainty), que tiene un efecto directo sobre los recursos hídricos.

Es necesario incorporar consideraciones sobre la incerteza del cambio climático en las etapas de planeamiento y toma de decisiones, y definir estrategias de adaptación a los impactos, para mejorar la eficiencia y resiliencia de las inversiones en programas, proyectos e infraestructura.

Como respuestas a los impactos del cambio climático ha sido propuesta una amplia variedad de estrategias de adaptación, que si bien pueden implementarse individualmente, han demostrado un mejor desempeño cuando se enmarcan en un conjunto planificado de medidas. (Hallegatte, 2009) sugiere algunos tipos de estrategias para lidiar con esa incerteza:

- Estrategias No-Regret: tienen justificación en sí mismas desde el punto de vista de la situación actual, aun si la amenaza climática específica no se concretara. Por ejemplo, políticas de uso del suelo destinadas a limitar la urbanización en áreas inundables; son en sí mismas necesarias, el contexto de cambio climático potencial las hace aún más convenientes.

- Estrategias Reversibles: las políticas de adaptación deben favorecer las estrategias de tipo reversible y flexible sobre las opciones irreversibles. El objetivo es minimizar el costo de los errores de predicción sobre el futuro cambio climático. Por ejemplo, la implementación de sistemas de alerta temprana, que pueden ajustarse periódicamente en función de nueva información.

- Estrategias de margen de seguridad: el sobredimensionamiento de la infraestructura, basado en criterios de diseño conservadores reduce su vulnerabilidad, a costos muy bajos o nulos. Por ejemplo, diseñar para el máximo impacto previsible.

- Estrategias Blandas: aumento de la eficiencia de la adaptación al cambio climático a partir de la implementación de herramientas institucionales o financieras. Son a su vez estrategias de tipo reversible.

- Estrategias que reducen los horizontes de toma de decisiones: una opción para limitar los costos asociados a la incerteza del cambio climático es la reducción de la vida útil de la infraestructura.

En este trabajo se realiza un análisis exploratorio del desempeño de ciertas estrategias de adaptación en escenarios de incerteza profunda, y se presentan algunos indicadores económicos que resultarían de la aplicación de cuatro alternativas diferentes en el drenaje urbano de la cuenca del arroyo Manzores (Figura 1), Concordia, Entre Ríos (Garat, 2017).

\section{METODOLOGÍA}

La metodología consistió en la simulación de una cuenca urbana para condiciones de lluvias extremas superiores a los valores actuales de proyecto. Las mismas se definieron en función de los impactos previsibles, según el estado actual del conocimiento (CIMA, 2015). Se utilizó el modelo hidrológico e hidrodinámico PCSWMM, desarrollado por la empresa canadiense Computacional Hydraulics International (CHI).

A partir de los resultados de las simulaciones se realizó el análisis económico (relación beneficiocosto $(\mathrm{B} / \mathrm{C})$ y Valor Presente Neto (VPN) de cuatro estrategias de adaptación, para distintas combinaciones de cambio climático previsto $\mathrm{y}$ realmente ocurrido.

Para ello, se implementaron los siguientes pasos metodológicos:

- Los sistemas de micro y macrodrenaje fueron proyectados para la tormenta de proyecto estacionaria PE (definida a partir de relaciones IDT actuales), para las recurrencias de diseño y verificación del sistema, considerando las condiciones futuras de urbanización.

- Posteriormente se analizó el desempeño hidráulico de esta red en tres escenarios hipotéticos de cambio 
climático, que cubren el rango presentado por indicadores estandarizados sugeridos por la (CIMA, 2015). En este análisis se utilizaron Comisión Europea.

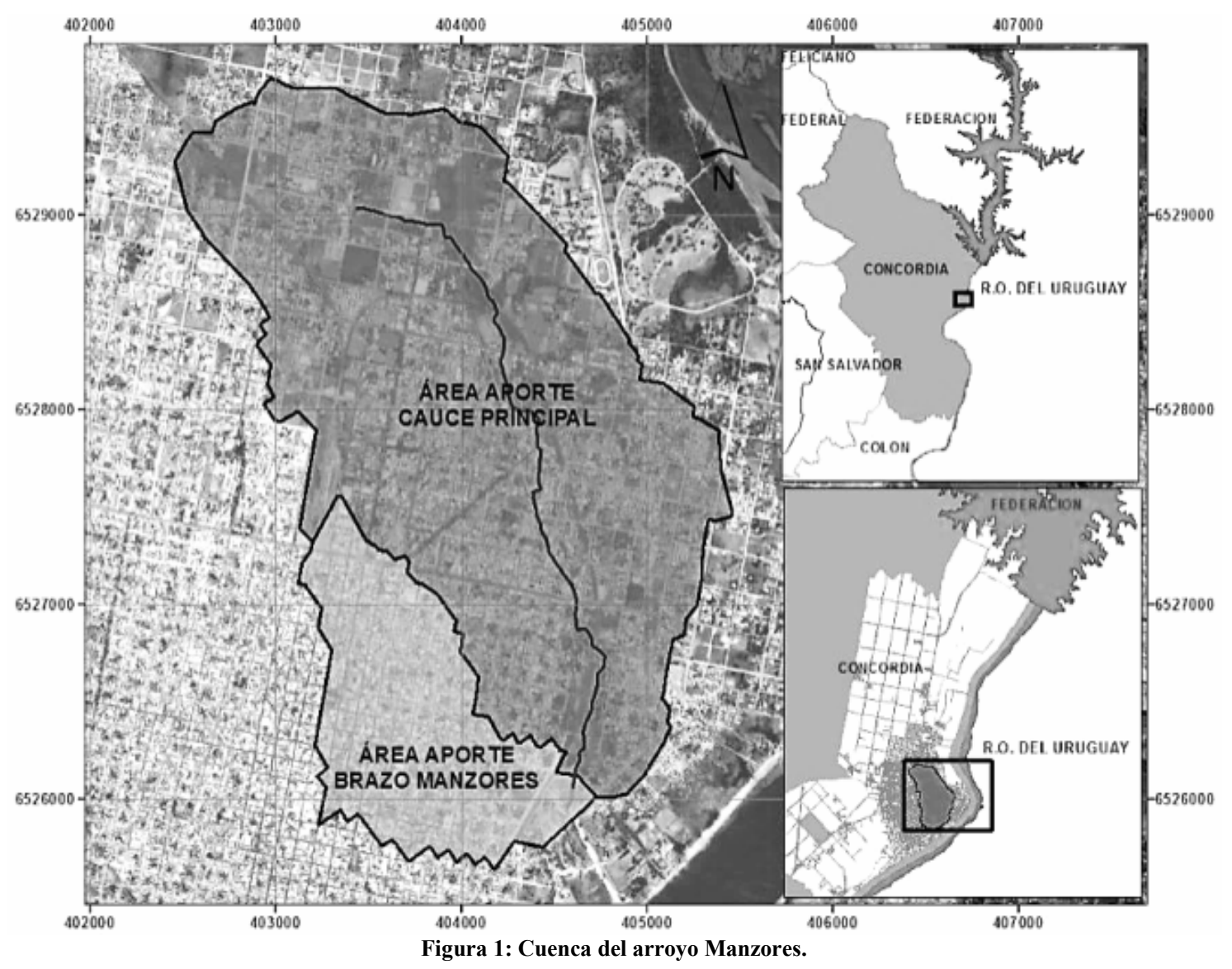

- Para el manejo del incremento potencial de las precipitaciones se definieron cuatro estrategias de planeamiento de los sistemas de drenaje urbano:

- El: Proyecto de la red para la tormenta estacionaria (estrategia de no acción),

- E2: Proyecto de la red para los máximos incrementos previstos en el fin del período de análisis,

- E3: Adecuación o reemplazo parcial/total de las obras,

- E4: Implementación de tecnologías LID (SWMM barrels).

- Para cada estrategia se calcularon los costos de implementación (como diferencia con respecto al costo de la estrategia E1), y los daños directos atribuibles a pérdidas por inundación de los inmuebles en las áreas afectadas, aplicando la metodología FLEMOps(+) (Thieken, 2008). Los beneficios económicos fueron estimados por la diferencia de daños entre las estrategias de planeamiento propuestas y la estrategia E1.

- Para la evaluación económica de las estrategias de planeamiento, se calcularon el valor presente neto VPN y la relación beneficio-costo $\mathrm{B} / \mathrm{C}$.

- En base a estos indicadores económicos, se efectuó un análisis exploratorio del desempeño de las estrategias de planeamiento en los escenarios hipotéticos considerados.

\section{Área de estudio}

La cuenca de estudio está ubicada en la planta urbana de la ciudad de Concordia, en la Provincia de Entre Ríos, Argentina. Tiene una superficie de 577,4Ha y corresponde al área de aportes del cauce principal del arroyo Manzores (Figura 2). 


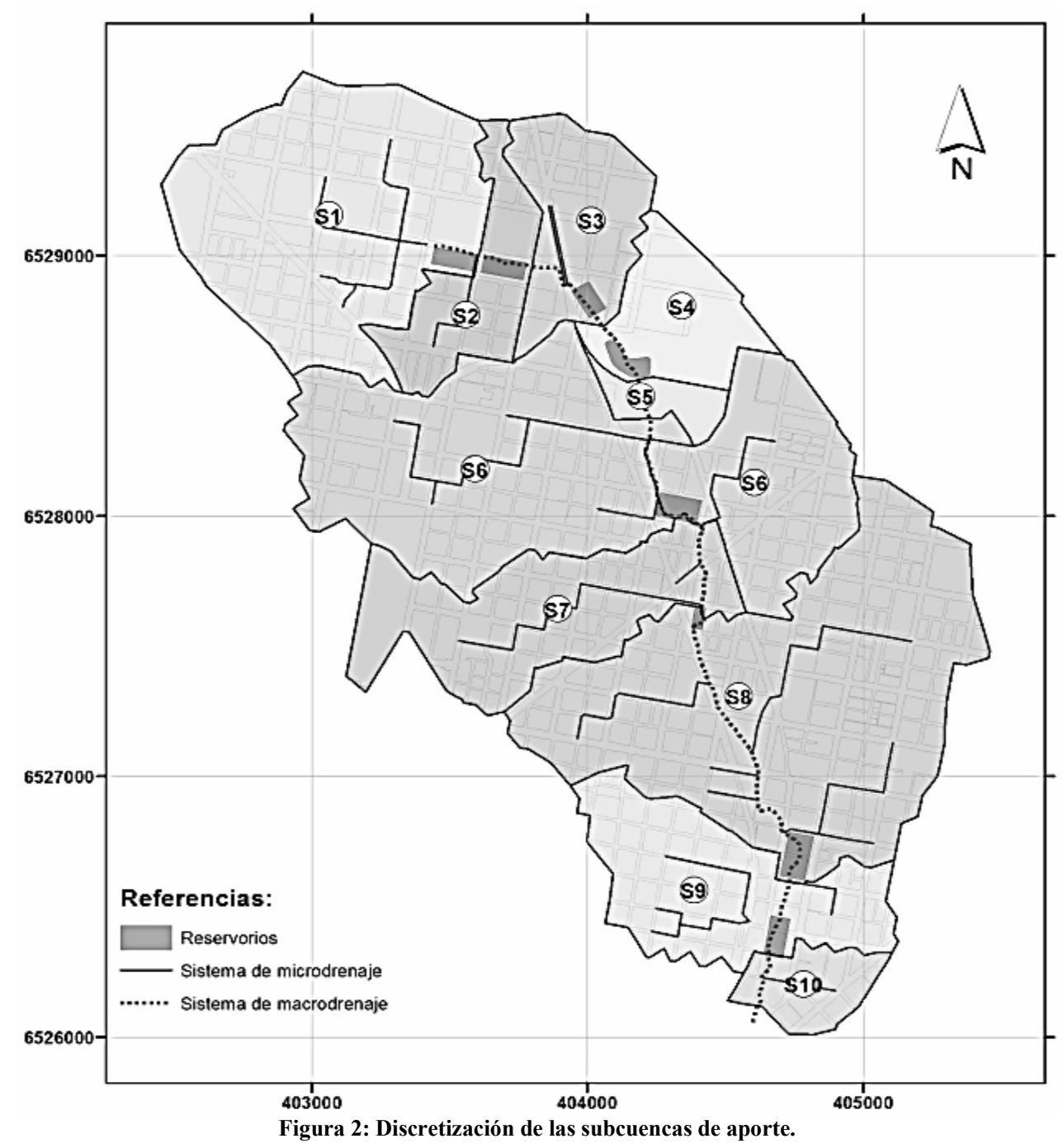

El canal principal de macrodrenaje se mantiene a cielo abierto y en estado seminatural, se desarrolla sobre una longitud de $3921 \mathrm{~m}$ hasta su desembocadura en el Río Uruguay. El Sistema de macrodrenaje incluye además nueve reservorios de detención de tipo "on line", materializados mediante excavaciones realizadas sobre el terreno natural, y confinados lateralmente por terraplenes.

El modelo de cuenca fue discretizada en 10 subcuencas de aporte a cada reservorio, desagregadas en 134 microcuencas de aporte a los colectores de desagües pluviales. Los factores actuales y futuros de ocupación de suelo se obtuvieron a partir del procesamiento de los archivos catastrales en formato vectorial y de los factores máximos de ocupación previstos por el Código de
Edificación del Municipio respectivamente, utilizando el software ARCGIS10.

La fracción permeable corresponde a la categoría $\mathrm{B}$ de la Clasificación Hidrológica del SCS, para la cual se adoptó valor $\mathrm{CN}=64$ (espacios abiertos con cobertura de pasto superiores al 75\%).

La red de drenaje está integrada por el sistema de microdrenaje (conductos subterráneos), el sistema de traslado superficial (calles) y la red de macrodrenaje (cauce principal, reservorios on line $y$ sus respectivas estructuras de descarga).

Para representar el comportamiento hidráulico del sistema ante eventos de diferente magnitud, se caracterizaron los siguientes escenarios, en función de su período de retorno $\mathrm{Tr}$ : 
- Escenario de proyecto del microdrenaje Tr: 5 años,

- Escenario de proyecto del macrodrenaje Tr: 25 años,

- Escenario de verificación del macrodrenaje Tr: 50 años.

El sistema de microdrenaje está conformado por 20 redes individuales de desagües pluviales, integradas por un total de 118 colectores subterráneos de hormigón armado. Se asumió un coeficiente de rugosidad de Manning del material constitutivo de la tubería $\mathrm{n}=0,014$, y un coeficiente de rugosidad equivalente $n=0,018$, ajustado para contemplar el efecto de pérdidas de carga singulares (UDFCD, 2008, pp.RO-30).

El sistema de calles hidráulicamente activas conduce el escurrimiento de las tormentas superiores a las de proyecto, que exceden la capacidad del sistema de conductos subterráneos. Está integrado 109 secciones, predominantemente de tipo local conforme a clasificación (UDFCD, 2008, pp.PL-17). Se utilizó una sección tipo equivalente, de $8 \mathrm{~m}$ de ancho y veredas de $3 \mathrm{~m}$, con cordones de $0,20 \mathrm{~m}$ de altura.

Para contemplar la presencia de obstrucciones, vehículos estacionados, basura, etc, se asumió un coeficiente de rugosidad equivalente de las calles $\mathrm{n}=0,030$ y 0,040 para las veredas.

Se consideraron tirantes máximos admisibles sobre el fondo de la cuneta de $0,20 \mathrm{~m}$ para el evento de proyecto, y de $0,45 \mathrm{~m}$ para el evento de verificación (UDFCD, 2008, pp.ST-6).

El sistema de macrodrenaje incluye 29 secciones transversales representativas del cauce principal y llanura de inundación del arroyo.

La geometría fue simplificada asumiendo para cada tramo una sección compuesta equivalente integrada por tres niveles (Figura 3), cuyas dimensiones se definieron para conducir el caudal pico del escenario de proyecto de las simulaciones (PE).

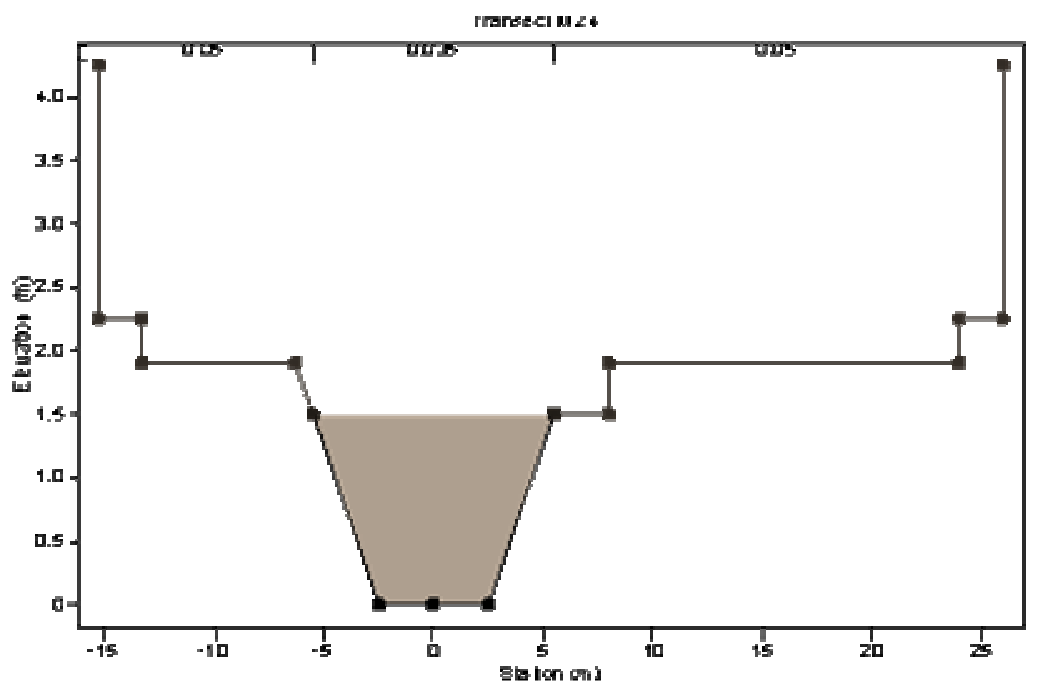

Figura 3: Sección trapezoidal equivalente de los tramos del macrodrenaje.

Se adoptó para el cauce principal una sección transversal trapecial, de taludes laterales variables entre $2 \mathrm{H}: 1 \mathrm{~V}$ y $4 \mathrm{H}: 1 \mathrm{~V}$ funcionando a sección llena para $\mathrm{Tr}=5$ años. Para las recurrencias de diseño del Macrodrenaje (Tr: 25 años) y de verificación del Macrodrenaje (Tr: 50 años) se consideraron dos niveles horizontales de ancho equivalente a la llanura de inundación. Se adoptaron coeficientes de rugosidad $n=0,035$ para el cauce principal y $n=0,050$ para la llanura de inundación (Arcement \& Schneider, 1989).
Los reservorios se dimensionaron para reducir los caudales máximos de postdesarrollo a valores compatibles con el grado de impermeabilidad actual de la cuenca. Para la modelación se adoptaron predominantemente volúmenes simplificados de planta rectangular y paredes verticales. El área en planta fue sujeta a ajustes sucesivos hasta alcanzar la regulación requerida para los escenarios de proyecto y verificación.

Las obras de descarga previstas consisten en orificios, vertederos de operación y vertederos de 
emergencia, diseñados para funcionar en rangos de profundidad determinados para cada escenario:

Los orificios, ubicados en el nivel inferior de los reservorios regulan los caudales de salida a tasas compatibles con las condiciones de urbanización actual para una recurrencia de 5 años (escenario de proyecto del microdrenaje).

Los vertederos de operación regulan los caudales a tasas compatibles con las condiciones de urbanización actual en la recurrencia de proyecto del macrodrenaje (Tr: 25 años). Se adoptaron vertederos rectangulares, de longitud variable entre 12 y 15 metros. Los vertederos de emergencia, se dimensionaron para descargar con seguridad los excesos sobre los caudales de diseño del macrodrenaje, evitando así eventuales daños estructurales originados por el desborde sobre los terraplenes de cierre de los reservorios. Su geometría se ajustó para el período de retorno de verificación del macrodrenaje (Tr: 50 años), adoptando vertederos rectangulares de longitud comprendida entre 10 y $15 \mathrm{~m}$.

\section{Simulación de los escenarios}

\section{Escenario de precipitación estacionaria (PE)}

Se adoptaron tormentas de diseño de 24 horas de duración, obtenidas mediante el Método de bloques alternos.

La relación intensidad-duración-recurrencia de tipo Sherman de la localidad de Concordia responde a la Ecuación (1) (Zamanillo et al, 2008):

$$
i=\frac{652,4 T_{r}^{0,26}}{(d+5)^{0,71}}
$$

donde, $\mathrm{i}$ = intensidad de lluvia en $\mathrm{mm} / \mathrm{h}$, $\mathrm{Tr}=$ período de retorno en años, $\mathrm{d}=$ duración de la tormenta en minutos.

\section{Escenarios de cambio climático}

Para evaluar los impactos del cambio climático se consideraron incrementos hipotéticos de la tormenta de proyecto estacionaria. Esta aproximación es similar a la utilizada por (Waters et al, 2003).

Se definieron tres escenarios hipotéticos de crecimiento de la precipitación máxima, designados como escenarios de cambio climático 1, 2 y 3, asumiendo que al final de un horizonte de planeamiento de 90 años (2013-2103) los mismos alcanzan los siguientes incrementos máximos:

- Escenario de cambio climático 1: 1,10PE

- Escenario de cambio climático 2: 1,20PE

- Escenario de cambio climático 3: 1,30PE

Para analizar el impacto a corto, mediano y largo plazo, se consideraron horizontes temporales de 30 años, correspondientes a los años 2043, 2073 y 2103. El crecimiento de la precipitación durante el período de análisis 2013-2103 se aproximó mediante tres incrementos de magnitud constante, uniformemente distribuidos durante los períodos analizados.

La evolución temporal de cada escenario hipotético de cambio climático se indica en laTabla 1.

Tabla 1. Secuencia temporal de los escenarios hipotéticos de

\begin{tabular}{|c|c|c|c|c|}
\hline \multirow{2}{*}{\begin{tabular}{|l|} 
Escenario \\
de cambio \\
climático
\end{tabular}} & \multirow[b]{2}{*}{$\begin{array}{c}\text { Incremento } \\
\text { máximo }\end{array}$} & \multicolumn{3}{|c|}{ Período de análisis } \\
\hline & & $\begin{array}{l}(2013- \\
2043)\end{array}$ & $\begin{array}{l}(2043- \\
2073)\end{array}$ & $\begin{array}{c}(2073- \\
2103)\end{array}$ \\
\hline 1 & $1,10 \mathrm{PE}$ & $1,03 \mathrm{PE}$ & $1,06 \mathrm{PE}$ & $1,10 \mathrm{PE}$ \\
\hline 2 & $1,20 \mathrm{PE}$ & $1,06 \mathrm{PE}$ & $1,13 \mathrm{PE}$ & $1,20 \mathrm{PE}$ \\
\hline 3 & $1,30 \mathrm{PE}$ & $1,10 \mathrm{PE}$ & $1,20 \mathrm{PE}$ & $1,30 \mathrm{PE}$ \\
\hline
\end{tabular}

\section{Estrategias de planeamiento adoptadas}

\section{Estrategia E1 (no acción)}

Se simula el funcionamiento de la red de drenaje para las dimensiones determinadas en el escenario estacionario, aceptando una disminución gradual del nivel de servicio ante incrementos hipotéticos generados por el cambio climático.

La tormenta de proyecto para el dimensionamiento de los componentes de los sistemas de micro y macrodrenaje corresponde a la precipitación estacionaria PE, según se indica en la Figura 4 para el escenario de cambio climático 2. El diseño de las obras permanece invariable en los tres escenarios de cambio climático.

\section{Estrategia E2 (precipitación máxima futura)}

Consiste en diseñar la red de drenaje en base a las máximas precipitaciones previstas para el fin de su vida útil, con una carga financiera asumida en el presente. Implica redimensionar la red para los tres escenarios de cambio climático. El redimensionamiento incluye a los colectores de microdrenaje, cauce principal y obras de descarga de los reservorios, asignando a cada diseño la siguiente designación: 
- E2-1 (Estrategia E2, escenario de cambio climático 1): la red de drenaje se proyecta para 1,10PE.

- E2-2 (Estrategia E2, escenario de cambio climático 2): la red se proyecta para 1,20PE.

- E2-3 (Estrategia E2, escenario de cambio climático 3): la red de drenaje se proyecta para 1,30PE.

La Figura 5 presenta la tormenta de proyecto de la estrategia E2-2 (escenario de cambio climático 2), corresponde a un incremento máximo de 1,20PE al final del período de análisis.

Estrategia E3 (reemplazo parcial de las obras) Los sistemas se proyectan y construyen para caudales correspondientes al corto plazo, contemplando el reemplazo parcial/total de la infraestructura cuando los incrementos generados por el cambio climático lo requieren.
Implica el eventual redimensionamiento de la red para cada corte temporal de los tres escenarios de cambio climático, que incluye a los colectores de microdrenaje, cauce principal y obras de descarga de los reservorios, asignando a cada diseño la siguiente designación:

- E3-1 (estrategia E3, escenario de cambio climático 1): las dimensiones de la red se ajustan a 1.03PE, 1,06PE y $1,10 \mathrm{PE}$ a corto, mediano y largo plazo respectivamente.

- E3-2 (estrategia E3, escenario de cambio climático 2 ): las dimensiones se ajustan a 1.06PE, 1,13PE y 1,20 PE a corto, mediano y largo plazo (según se indica en la Figura 6).

- E3-3 (estrategia E3, escenario de cambio climático 3 ): las dimensiones se ajustan a 1.10PE, 1,20PE y 1,30 PE.

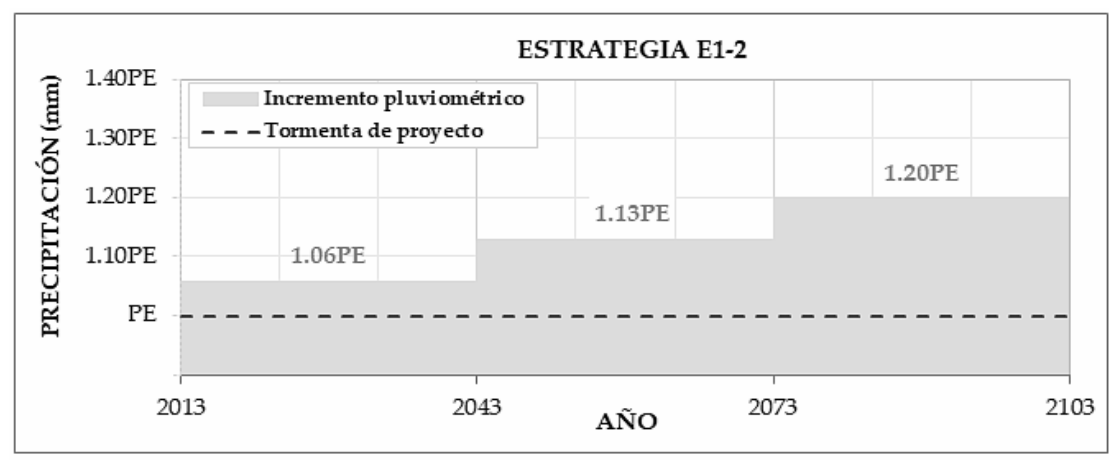

Figura 4: Estrategia E1 -Proyecto de la red para la precipitación estacionaria.

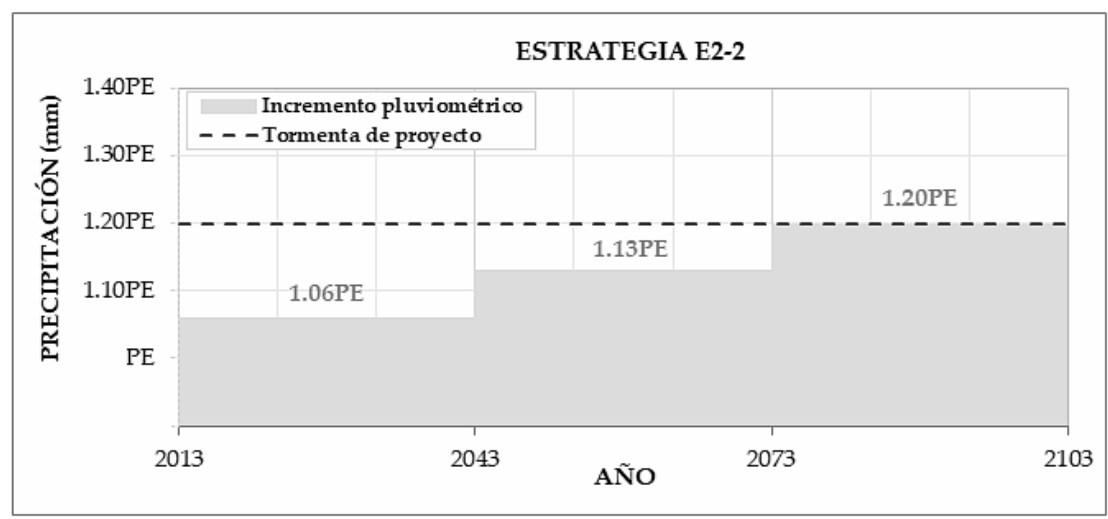

Figura 5: Estrategia E2-2 -Proyecto de la red para precipitaciones futuras

\section{Estrategia E4 (alternativa mixta)}

La estrategia E4 consiste en una alternativa de planeamiento mixta, que combina los siguientes criterios:

- Proyecto de los conductos de microdrenaje para la precipitación PE correspondiente al contexto climático estacionario (criterio de proyecto de la estrategia E1).

- Control del escurrimiento de las superficies impermeables mediante tanques de almacenamiento domiciliarios.

- Redimensionamiento del sistema de macrodrenaje para los incrementos previstos en los tres 
horizontes temporales de cada escenario de cambio climático (criterio de proyecto de la estrategia E3).

Para regular los incrementos de cada escenario de cambio climático se asumieron tres grados diferentes de control (cobertura espacial de los tanques de almacenamiento). Esta regulación se obtuvo asumiendo coberturas del $25 \%, 50 \%$ y $75 \%$ de viviendas abastecidas con tanques para los escenarios 1,10PE, 1,20PE y 1,30PE respectivamente. La Figura
7 presenta la tormenta de proyecto de la estrategia E4-2 (escenario de cambio climático 2).

Se consideró la utilización de dispositivos de almacenamiento domiciliarios desarrollados en la Regional Litoral del Instituto Nacional del Agua (INA). Los mismos fueron simulados mediante la incorporación de controles LID (Low Impact Development) de tipo Rain Barrel (tanque o cisterna, en adelante RB) al modelo de cuenca PCSWMM.

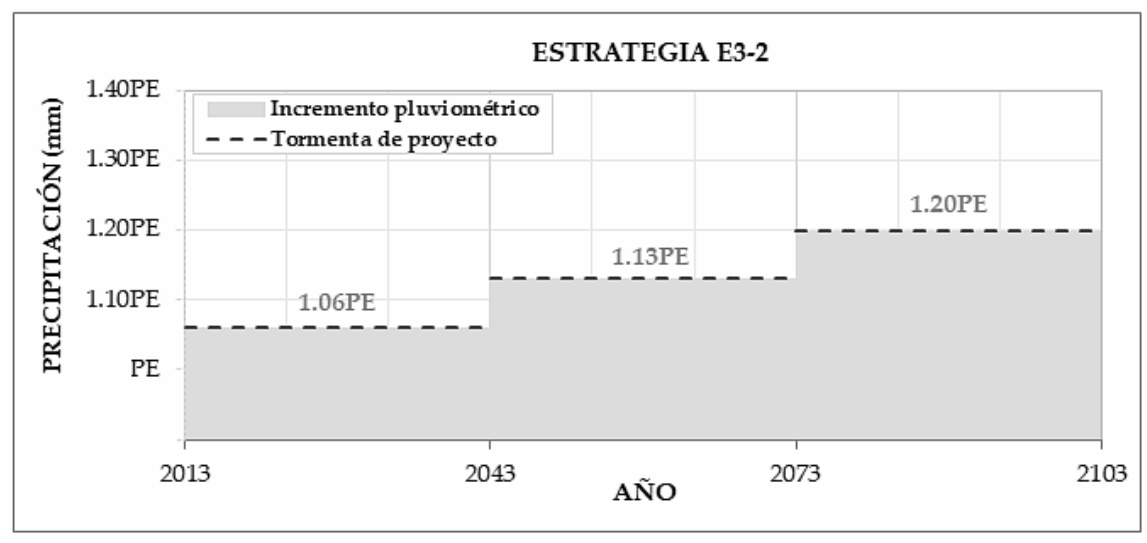

Figura 6: Estrategia E3-2 -Reemplazo parcial de las obras.

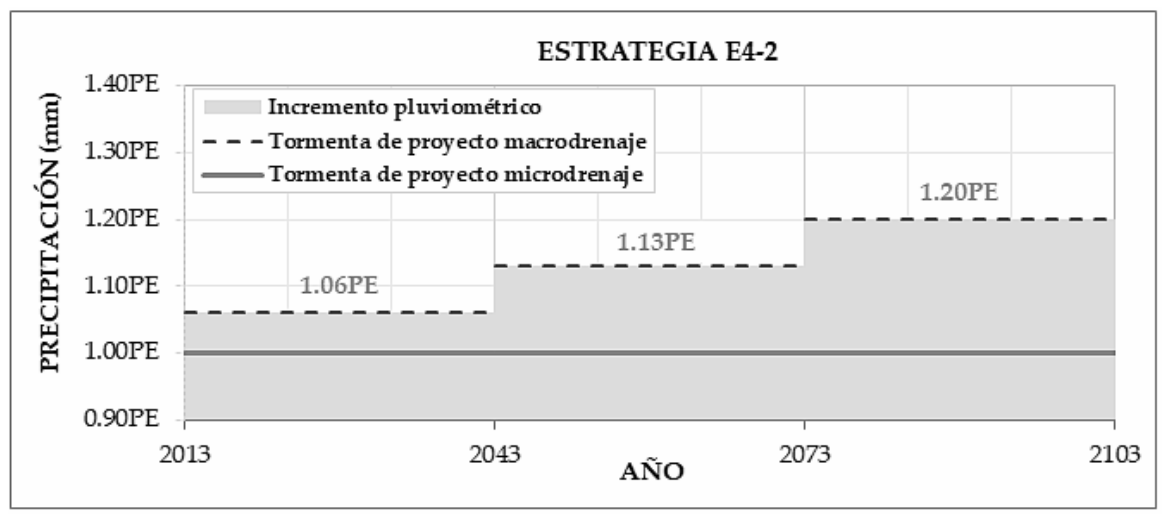

Figura 7: Estrategia E4-2 - Alternativa mixta.

\section{Daños por inundación}

Para estimar las pérdidas directas por anegamiento de viviendas en escenarios de cambio climático se utilizó la metodología FLEMOps ("Flood Loss Estimation MOdel for the private sector") (Thieken et al., 2007; Thieken, 2008).

Este modelo se basa en datos empíricos de 1697 hogares, afectados por inundaciones severas en Alemania durante 2002, 2005 y 2006, donde se recopilaron grandes conjuntos de datos sobre pérdidas por inundaciones y los factores involucrados mediante entrevistas telefónicas a hogares y empresas. Junto con los datos de encuestas, que evaluaron los daños a la infraestructura, se utilizaron las experiencias y datos de inundaciones recientes para obtener y evaluar nuevos modelos de pérdida para los distintos sectores.

El modelo estima las pérdidas directas adimensionales como un porcentaje del valor de la edificación (Figura 8) en función del nivel de agua (cinco categorías), tipo de construcción (tres clases: unifamiliar, vivienda adosada y multifamiliar) y calidad de la edificación (media/baja y alta) según se indica en la Tabla 2. 
El modelo considera también los efectos de medidas de precaución privadas y de la contaminación del agua de inundación, mediante factores de escala que se resumen en la Tabla 3.

Para la implementación del Modelo FLEMOps(+) (Thieken, 2008) se asumieron los siguientes parámetros: vivienda multifamiliar, calidad de las construcciones media, condiciones de contaminación medias por conexión a desagües cloacales a la red pluvial, y grado de precaución nulo.
El monto total atribuible a pérdidas directas por inundación se obtuvo multiplicando el valor total de los inmuebles afectados por las relaciones adimensionales estimadas en cada tramo de la red

Se determinó la superficie edificada de los inmuebles potencialmente susceptibles a la inundación, discriminándolas en sectores correspondientes a cada colector de microdrenaje para lo cual se procesó la información suministrada por la Dirección de Catastro del Municipio.

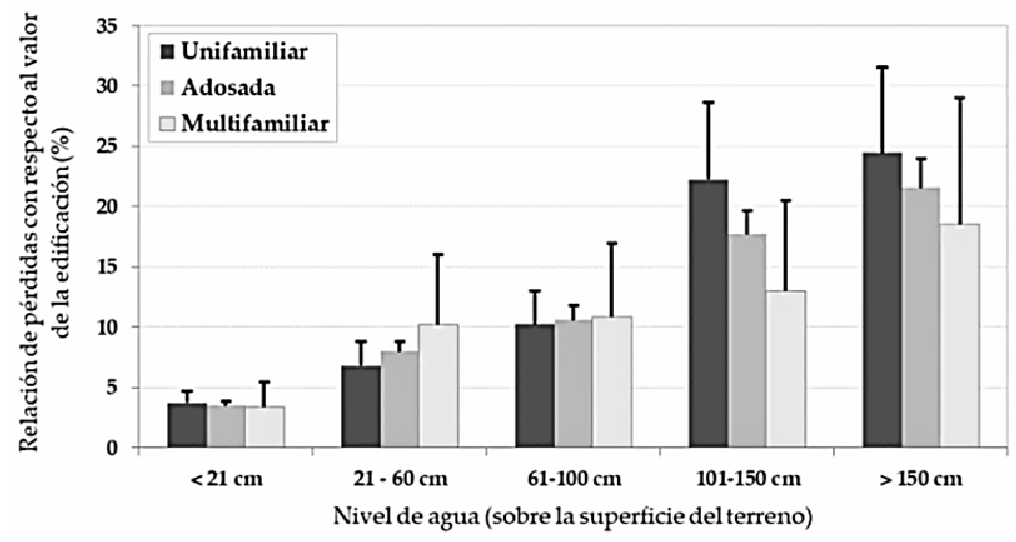

Figura 8: Modelo de microescala FLEMOps.

Tabla 2. Modelo de microescala FLEMOps.

\begin{tabular}{|c|c|c|c|c|c|c|}
\hline \multirow{2}{*}{$\begin{array}{c}\text { Nivel de agua } \\
\text { (cm) }\end{array}$} & \multicolumn{3}{|c|}{ Calidad edificación media/baja } & \multicolumn{3}{c|}{ Calidad de edificación alta } \\
\cline { 2 - 7 } & $\begin{array}{c}\text { Uni } \\
\text { familiar }\end{array}$ & $\begin{array}{c}\text { Vivienda } \\
\text { adosada }\end{array}$ & $\begin{array}{c}\text { Multi } \\
\text { familiar }\end{array}$ & $\begin{array}{c}\text { Uni } \\
\text { familiar }\end{array}$ & $\begin{array}{c}\text { Vivienda } \\
\text { adosada }\end{array}$ & $\begin{array}{c}\text { Multi } \\
\text { familiar }\end{array}$ \\
\hline$<21$ & 3,70 & 3,50 & 3,40 & 4,70 & 3,80 & 5,40 \\
$21-60$ & 6,80 & 8,00 & 10,20 & 8,80 & 8,80 & 16,00 \\
$61-100$ & 10,20 & 10,60 & 10,90 & 13,05 & 11,8 & 16,95 \\
$101-150$ & 22,25 & 17,70 & 13,05 & 28,60 & 19,65 & 20,50 \\
$>150$ & 24,45 & 21,55 & 18,50 & 31,50 & 23,95 & 29,00 \\
\hline
\end{tabular}

Tabla 3. Efectos de la contaminación y medidas de precaución FLEMOSps (+), 2008.

\begin{tabular}{|l|c|c|}
\hline Factores de escala para relaciones de pérdida & Edificación privada & Edificación comercial \\
\hline Sin contaminación, sin precaución & 0,92 & 1,02 \\
\hline Sin contaminación, precaución media & 0,64 & 0,82 \\
\hline Sin contaminación, precaución elevada & 0,41 & 0,67 \\
\hline Contaminación media, sin precaución & 1,2 & 1,28 \\
\hline Contaminación media, precaución media & 0,86 & 1,03 \\
\hline Contaminación media, precaución elevada & 0,71 & 0,84 \\
\hline Contaminación elevada, sin precaución & 1,58 & 1,28 \\
\hline Contaminación elevada, precaución media & - & 1,03 \\
\hline Contaminación elevada, precaución elevada & - & 0,84 \\
\hline
\end{tabular}


En el primer horizonte (H1) de 30 años del período de análisis (corto plazo 2013-2043) se utilizó la superficie edificada actual.

Se asumió que en el último horizonte H3 (largo plazo 2073-2103) las condiciones de urbanización corresponden al máximo factor de uso del suelo reglamentado por el Código de edificación.

En el intervalo medio (H2) del período de análisis (mediano plazo 2043-2073) se asumió la superficie edificada promedio entre ambas condiciones extremas.

Para cuantificar los daños asociados al sistema de macrodrenaje, se estimaron los desbordes causados por los incrementos de nivel del curso de macrodrenaje en cada escenario de cambio climático, definiendo dos tipos básicos de falla:

Desbordes del cauce principal: afecta a las propiedades ubicadas en la planicie de inundación. Se asumió que las viviendas se encuentran ubicadas en el segundo nivel de la planicie de inundación, en base a la información topográfica y relevamiento in situ de las áreas potencialmente afectadas.

Desborde de los reservorios: esta falla se produce en el escenario de verificación del macrodrenaje y representa una condición crítica del sistema. Afecta a un número significativo de propiedades en las áreas de influencia de los reservorios.

\section{Valor esperado de los daños}

En cada escenario de cambio climático se cuantificaron los daños por inundación de las redes de micro y macrodrenaje aplicando el Modelo FLEMOps(+). Para cada estrategia, se estimó el valor esperado de los daños (Expected Annual Damage EAD): aquel que podría esperarse que ocurra anualmente como consecuencia de la falla de la red de drenaje.

Estadísticamente representa la esperanza matemática del daño: el valor medio de la variable aleatoria continua $\mathrm{x}$ (daño anual por inundación), cuya función densidad de probabilidad es $\mathrm{f}(\mathrm{x})$ corresponde a la esperanza matemática $\mathrm{E}(\mathrm{x})$, indicada en la ecuación (2)

$$
E(x)=\int x f(x) d x
$$

El daño en un año cualquiera del período analizado puede interpretarse como la integral de la función densidad de probabilidad sobre todo el rango de probabilidades. De las diversas metodologías propuestas para desarrollar la solución de esta integral (Olsen, 2014), se adoptó la integración numérica por el método trapezoidal, ecuación (3)

$$
E A D=\frac{1}{2} \sum_{i=1}^{N}\left(\frac{1}{T r_{i}}-\frac{1}{T r_{i+1}}\right)\left(D_{i}+D_{i+1}\right)
$$

Siendo:

EAD: valor esperado de los daños (\$/año),

$\mathrm{N}$ : número de eventos,

$\operatorname{Tr}_{\mathrm{i}}$ y $\mathrm{Tr}_{\mathrm{i}+1}$ : períodos de retorno analizados (años),

$\mathrm{D}_{\mathrm{i}}$ y $\mathrm{D}_{\mathrm{i}+1}$ : daños provocados por la inundación en los eventos analizados $(\$)$,

EAD se obtuvo a partir de tres eventos $(\mathrm{N}=3)$, que corresponden a las recurrencias de proyecto $\mathrm{y}$ verificación del micro y macrodrenaje $(5,25$ y 50 años (Figura 9). La designación genérica (Figura 10) adoptada para identificar los tres escalones de las series de valor esperado de los daños es la siguiente:

- Corto plazo (2013-2043): EADa

- Mediano plazo (2043-2073): EADb

- Largo plazo (2073-2103): EADc

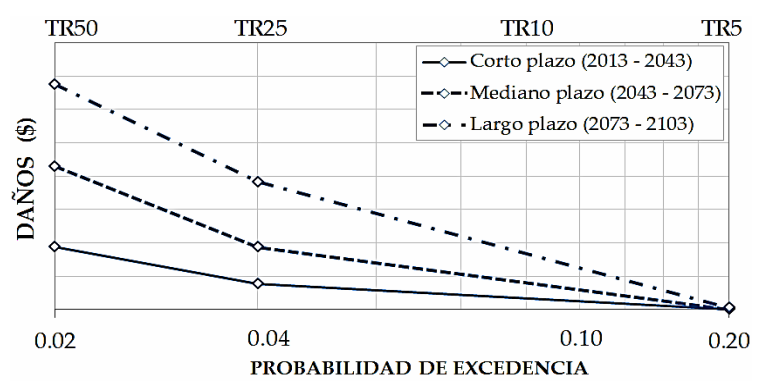

Figura 9: Relación Daños - Probabilidad de ocurrencia.

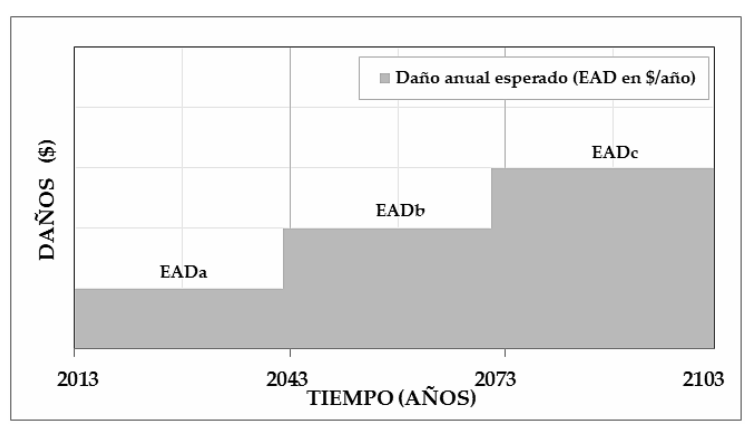

Figura 10: Valor esperado de los daños EAD (\$/año). 
La cuantificación del EAD implica la ejecución de 27 simulaciones para cada estrategia: cada escenario de cambio climático está integrado por tres escalones de precipitación máxima correspondientes a las secuencias (2013-2043), (2043-2073) y (20732103), y tres eventos de funcionamiento de la red (proyecto del microdrenaje (Tr: 5 años), proyecto del macrodrenaje (Tr: 25 años) y verificación del macrodrenaje (Tr: 50 años).

\section{Actualización de los daños futuros}

Para actualizar las tres series escalonadas de daños anuales (EADa, EADb y EADc) se utilizaron las fórmulas del valor presente de anualidades diferidas, que comprende dos etapas de actualización:

En la primera etapa se actualiza cada serie uniforme a su inicio relativo, determinando el daño anual esperado equivalente de la etapa i (Di en \$). Para ello se aplica el Factor de Actualización de una Serie uniforme (FAS), a una tasa de descuento $r$ según ecuación (4), siendo na=30 años el período de actualización de cada serie uniforme EADa, EADb y EADc. Los daños Di obtenidos con la ecuación (4) constituyen pérdidas concentradas en los años 2013, 2043 y 2073 del flujo de fondos, de magnitud equivalente a las series distribuidas.

$$
D i=E A D\left[\frac{(1+r)^{n a}-1}{i(1+r)^{n a}}\right]
$$

El Valor Presente de los Daños (VPD en \$) atribuibles a las pérdidas por se obtiene actualizando los daños Di de cada etapa al año base 2013, a través del Factor de actualización simple de acuerdo a ecuación (5)

$$
V P D=\sum_{i=1}^{i=n} D i\left[\frac{1}{(1+r)^{n a}}\right]
$$

\section{Actualización de los costos de construcción}

Los costos de construcción de las estrategias E1 y E2se ubican en el año inicial del período de análisis (año base: 2013).

En el caso de las estrategias E3 y E4, la inversión está integrada por un monto inicial (año base), y por inversiones parciales en horizontes temporales intermedios, designadas como $\mathrm{Ci}$ : costo de Construcción de la etapa i (en \$). Los mismos fueron actualizados al año base mediante el Factor Simple de Actualización (FSA), determinando el Valor Presente del Costo de construcción (VPC en \$) según ecuación (6).

$$
V P C=\sum_{i=1}^{i=n} C i\left[\frac{1}{(1+r)^{n a}}\right]
$$

Ci: costo de construcción de la etapa i (\$)

r: tasa de descuento na: período de actualización (años)

\section{Indicadores seleccionados}

Para evaluar el desempeño económico de las estrategias en cada escenario de cambio climático se seleccionaron los siguientes indicadores: relación costo-beneficio B/C y Valor Presente Neto.

En cada horizonte temporal se determinaron las relaciones costo - beneficio $\mathrm{B} / \mathrm{C}$ de las estrategias, definidas como el cociente entre el valor presente de los beneficios (B en \$) y el valor presente de los costos de implementación de las estrategias ( $\mathrm{C}$ en $\$$ ).

Los beneficios económicos de las estrategias de planeamiento E2, E3 y E4 son los costos netos evitados con respecto a la situación de no acción (E1). Se cuantificaron como la diferencia entre el valor presente de los daños acumulados (VPD en \$) de la estrategia E1 y el VPD de cada estrategia para cada horizonte temporal de los tres escenarios de cambio climático. Los costos de implementación de cada estrategia se calcularon como la diferencia entre los valores actualizados de sus costos de construcción (VPC en \$), y el correspondiente a la estrategia E1.

El valor presente neto (VPN en \$) de cada estrategia de planeamiento, se determinaron a partir de la sumatoria de los costos actualizados al año base 2013, según Ecuación (7).

$$
V P N=V P C+V P D
$$

$$
V P N=\sum_{i=1}^{i=n} C i\left[\frac{1}{(1+r)^{n a}}\right]+\sum_{i=1}^{i=n} D i\left[\frac{1}{(1+r)^{n a}}\right]
$$

La tasa de descuento seleccionada para el análisis económico de las estrategias es $\mathrm{r}=4 \%$, ubicada en el rango acordado en las reuniones de coordinación de la CEPAL, validadas por los integrantes de equipos 
nacionales y paneles de asesores sobre cambio climático (CEPAL, 2014, p.60).

\section{Combinatoria de impactos previstos y reales}

En escenarios de incerteza profunda, el incremento de las precipitaciones máximas (impacto previsto) está sujeto a potenciales alteraciones (impacto real). Es decir que la evolución futura de las precipitaciones máximas puede generar incrementos o reducciones de los beneficios de cada estrategia con respecto a los valores de referencia, que modifican los resultados de la evaluación económica.

En la Figura 11 se presenta el resumen de escenarios analizados para evaluar la variación del desempeño económico de las estrategias en escenarios de incerteza severa. Las diagonales sombreadas representan los escenarios de cambio climático para los cuales fueron proyectadas las redes respectivas (impacto previsto=impacto real).

En las celdas ubicadas sobre la diagonal principal se ubican las redes sobredimensionadas: escenarios de menor incremento de la precipitación máxima que el asumido en el proyecto de la red (impacto real menor al impacto previsto). Esta condición implica una reducción de los daños a expensas de un costo de construcción mayor.

En las celdas ubicadas bajo la diagonal principal se encuentran las redes subdimensionadas: escenarios de mayor incremento de la precipitación máxima que el asumido en el proyecto de la red (impacto real mayor al impacto previsto). Esta condición implica una reducción del costo de construcción a expensas de un incremento de los daños.

\begin{tabular}{|c|c|c|c|c|c|c|c|c|c|}
\hline \multirow{2}{*}{$\begin{array}{c}\text { Escenario de } \\
\text { cambio climático }\end{array}$} & \multicolumn{3}{|c|}{ Estrategia E2 } & \multicolumn{3}{|c|}{ Estrategia E3 } & \multicolumn{3}{|c|}{ Estrategia E4 } \\
\hline & E2-1 & E2-2 & E2-3 & E3-1 & E3-2 & E3-3 & E4-1 & E4-2 & E4-3 \\
\hline 1 & E2-1 & E2-2-1 & E2-3-1 & E3-1 & E3-2-1 & E3-3-1 & E4-1 & E4-2-1 & E4-3-1 \\
\hline 2 & E2-1-2 & E2-2 & E2-3-2 & E3-1-2 & E3-2 & E3-3-2 & E4-1-2 & E4-2 & E4-3-2 \\
\hline 3 & E2-1-3 & E2-2-3 & E2-3 & E3-1-3 & E3-2-3 & E3-3 & E4-1-3 & E4-2-3 & E4-3 \\
\hline
\end{tabular}

Figura 11: Combinatoria de impactos de cambio climático, previstos en el proyecto y realmente acontecidos.

\section{RESULTADOS Y DISCUSIÓN}

\section{Indicadores de impacto del cambio climático}

Los indicadores utilizados son: el porcentaje de calles afectadas, el tirante de agua en las calles y el caudal máximo en el curso de macrodrenaje (Arroyo Manzores).

De acuerdo a la magnitud del tirante de agua (h) en las calles se adoptó la siguiente clasificación:

- Calles hidráulicamente activas: $0<\mathrm{h}<0,20 \mathrm{~m}$

- Calles anegadas:0,20 $\leq \mathrm{h}<0,50 \mathrm{~m}$

- Calles críticas: $h \geq 0,50 \mathrm{~m}$

\section{Proyecto del microdrenaje $(\mathrm{Tr}=5$ años $)$}

En el escenario de proyecto del microdrenaje (PE5) los conductos funcionan a gravedad y no transfieren caudales al sistema superficial, es decir que la cantidad de calles hidráulicamente activas es nula.
La Tabla 4 resume los impactos asociados a cada escenario de cambio climático:

La cantidad de calles hidráulicamente activas se incrementa entre 2 y $35 \%$, con tirantes variable (mediana entre 7 y $20 \mathrm{~cm}$ ).

Los resultados de las simulaciones indican la aparición de tirantes superiores al cordón de vereda, condición que ha sido designada como anegamiento de calles, que afectan porcentajes variables entre 2 y $6 \%$ de la longitud total de las calles.

Tabla 4. Indicadores de impacto $\operatorname{Tr} 5$ años.

\begin{tabular}{|c|c|c|c|}
\hline \multicolumn{1}{|c|}{ Tabla 4. Indicadores de impacto Tr 5 años. } \\
\hline Escenario & $\begin{array}{c}\text { Inundación } \\
\text { calles }\end{array}$ & $\begin{array}{c}\text { Mediana } \\
\text { tirantes }\end{array}$ & $\begin{array}{c}\text { Qmáx } \\
\text { Manzores }\end{array}$ \\
\hline $1,10 \mathrm{PE}$ & $2 \%$ & $7 \mathrm{~cm}$ & $+18 \%$ \\
\hline $1,20 \mathrm{PE}$ & $17 \%$ & $15 \mathrm{~cm}$ & $+32 \%$ \\
\hline $1,30 \mathrm{PE}$ & $35 \%$ & $20 \mathrm{~cm}$ & $+48 \%$ \\
\hline
\end{tabular}

En todos los escenarios de cambio climático el incremento de niveles en los reservorios genera la 
entrada en funcionamiento de los vertederos de operación V3 a V9, previstos para operar recién en la recurrencia de proyecto del macrodrenaje (PE25).

\section{Proyecto del macrodrenaje (Tr: 25 años)}

En los tres escenarios de cambio climático no se generan aumentos significativos de los tirantes máximos. El principal impacto es el crecimiento del número de tramos afectados.

La longitud de calles anegadas tiene incrementos comprendidos entre el $14 \%$ y $39 \%$ con respecto al escenario de precipitación estacionaria (PE25).

El porcentaje de calles críticas se incrementa entre $1 \%$ y $7 \%$ con respecto al escenario de precipitación estacionaria (Tabla 5).

Tabla 5. Indicadores de impacto Tr 25 años.

\begin{tabular}{|c|c|c|c|}
\hline Escenario & $\begin{array}{c}\text { Calles } \\
\text { anegadas }\end{array}$ & $\begin{array}{c}\text { Calles } \\
\text { críticas }\end{array}$ & $\begin{array}{c}\text { Qmáx } \\
\text { Manzores }\end{array}$ \\
\hline $1,10 \mathrm{PE}$ & $14 \%$ & $1 \%$ & $+18 \%$ \\
\hline $1,20 \mathrm{PE}$ & $25 \%$ & $2 \%$ & $+40 \%$ \\
\hline $1,30 \mathrm{PE}$ & $39 \%$ & $7 \%$ & $+48 \%$ \\
\hline
\end{tabular}

La mediana de los incrementos de caudal $\Delta \mathrm{Q}(\%)$ en los tramos del macrodrenaje crece entre un $18 \%$ y $48 \%$. Esto causa la entrada en funcionamiento de los vertederos de emergencia, previstos para funcionar recién en el evento de verificación del macrodrenaje. Los desbordes en la sección media equivalente del arroyo alcanzan tirantes entre $0,18 \mathrm{~m}$ y $0,44 \mathrm{~m}$ sobre el segundo nivel de la planicie inundable, afectando longitudes variables entre el $96 \%$ y la totalidad de los tramos del macrodrenaje.

\section{Verificación del macrodrenaje (Tr: 50 años)}

En los escenarios de cambio climático analizados, los tirantes máximos no presentan incrementos sustanciales, y el principal impacto es el aumento del número de tramos afectados.

Según se indica en la Tabla 6, la longitud de calles anegadas se incrementa entre un $15 \%$ y un $38 \%$. El porcentaje de calles críticas aumenta entre $6 \%$ y $22 \%$. La mediana de los incrementos del caudal máximo varía entre $9 \%$ y $17 \%$.

La peligrosidad potencial de este escenario se asocia a la probabilidad de colapso de los reservorios por desbordes, que afectarían áreas de gran extensión superficial y bajo tirante.

Tabla 6. Indicadores de impacto $\operatorname{Tr} 50$ años.

\begin{tabular}{|c|c|c|c|}
\hline Escenario & $\begin{array}{c}\text { Calles } \\
\text { anegadas }\end{array}$ & $\begin{array}{c}\text { Calles } \\
\text { críticas }\end{array}$ & $\begin{array}{c}\text { Qmáx } \\
\text { Manzores }\end{array}$ \\
\hline $1,10 \mathrm{PE}$ & $15 \%$ & $6 \%$ & $+9 \%$ \\
\hline $1,20 \mathrm{PE}$ & $30 \%$ & $15 \%$ & $+16 \%$ \\
\hline $1,30 \mathrm{PE}$ & $38 \%$ & $22 \%$ & $+17 \%$ \\
\hline
\end{tabular}

Para cada escenario hipotético de cambio climático se calcularon los volúmenes de inundación $\mathrm{Vi}\left(\mathrm{m}^{3}\right)$ generados por los desbordes. Estos volúmenes se expresaron en términos relativos, como porcentaje del volumen de cada reservorio $\mathrm{Vr}$.

Los volúmenes relativos $\operatorname{Vi} / \operatorname{Vr}(\%)$ se comparan en la Figura 12 y Tabla 7, donde puede observarse que debido a su escasa capacidad de almacenamiento uno de los reservorios (R7) constituye un punto crítico de la red, exponiendo a las áreas adyacentes a la mayor amenaza en todos los escenarios de cambio climático. Dependiendo del evento, el volumen de los desbordes en este reservorio varía entre el $35 \%$ y $243 \%$ del volumen total.

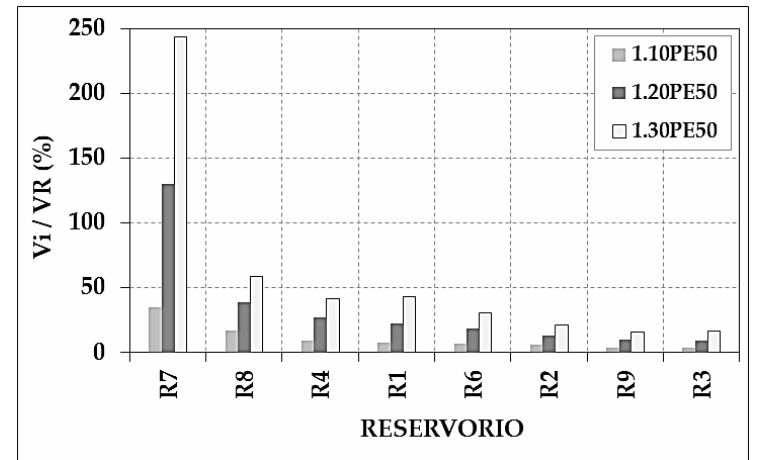

Figura 12: Desbordes en reservorios (Vi/Vr en \%).

Tabla 7: Volumen desbordes en reservorios Vi/VR (en \%).

\begin{tabular}{|c|c|c|c|c|c|c|c|c|}
\hline Reservorio & R7 & R8 & R4 & R1 & R6 & R2 & R9 & R3 \\
\hline 1.10PE50 & 35 & 17 & 9 & 8 & 7 & 6 & 4 & 4 \\
\hline 1.20PE50 & 130 & 39 & 27 & 23 & 18 & 13 & 10 & 9 \\
\hline 1.30PE50 & 243 & 59 & 42 & 43 & 31 & 21 & 16 & 16 \\
\hline
\end{tabular}

Los desbordes previstos en los restantes reservorios, se ordenaron de acuerdo a la magnitud prevista del impacto. Según se indica en la Tabla 7 , su magnitud es variable de acuerdo al reservorio y magnitud del cambio climático, desde un mínimo del $4 \%$ hasta un $59 \%$ del volumen total. 


\section{Evaluación económica de las estrategias}

Se utilizó la metodología de análisis BeneficioCosto para los 3 horizontes temporales futuros $(\mathrm{Hl}$ 2013-2043; H2, 2013-2073 y H3, 2013-2103) de los escenarios de cambio climático. Para ello se definieron los beneficios como costos netos evitados por implementación de cada estrategia con respecto a la situación de no acción (El), y los costos de implementación como las diferencias de valor presente del costo de construcción con respecto a la situación de no acción (El).

Se utilizaron los análisis de precios (valores correspondientes al año 2013) suministrados por la Comisión Administradora para el Fondo Especial de Salto Grande (CAFESG) y por la Unidad Ejecutora Provincial de Entre Ríos (UEP).

\section{Línea de base estacionaria}

La línea de base estacionaria representa los daños generados por la tormenta de proyecto de magnitud $\mathrm{PE}$, que aún en las condiciones actuales genera daños directos a las edificaciones.

Posteriormente, se consideraron las pérdidas generadas en escenarios de impacto leve $(1,10 \mathrm{PE})$, moderado $(1,20 \mathrm{PE})$ y extremo $(1,30 \mathrm{PE})$ de cambio climático, para cuantificar la diferencia de daños con respecto a la condición actual durante el período de tiempo analizado.
Para la definición de la línea de base (LB-PE) se asumió que la tormenta de proyecto de magnitud $\mathrm{PE}$ es constante durante todo el horizonte de planeamiento. Como consecuencia, los tirantes de agua en las edificaciones, áreas urbanizadas del cauce principal y áreas ubicadas en las zonas de influencia de los reservorios expuestas al riesgo de inundación no se modifican con el transcurso del tiempo.

A partir de los resultados de las simulaciones y la posterior cuantificación de los daños se estima que el valor presente de las pérdidas varía en un orden comprendido entre $\$ 23.500 .000$ y $\$ 43.150 .000$ en función del escenario de cambio climático.

Para evaluar la distribución espacial de las pérdidas el valor presente total de los daños VPD fue desagregado en cinco Áreas de Daño (AD), cuya incidencia media porcentual se indica en la Figura 13.

El mayor impacto económico está previsto en las áreas de daño $\mathrm{AD} 2$ y $\mathrm{AD} 3$, que en promedio representan un $45 \%$ y $35 \%$ del daño total. Las áreas $\mathrm{AD} 1$ y AD4 representan en promedio un $10 \%$ de las pérdidas totales, mientas que el área AD5 solo constituye un $1 \%$ del valor total de los daños.

La composición del valor presente de los daños está representada en la Figura 14 donde se indica el aporte individual (en \$) de cada área $(\mathrm{AD})$ a la línea de base total.

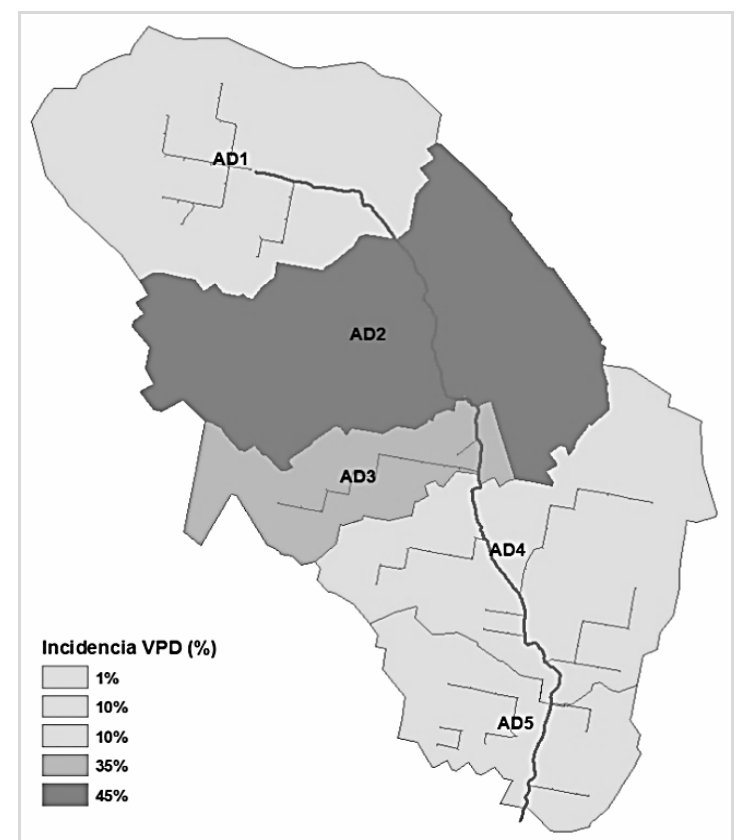

Figura 13: Incidencia porcentual en el VPD de las áreas de daños AD. 


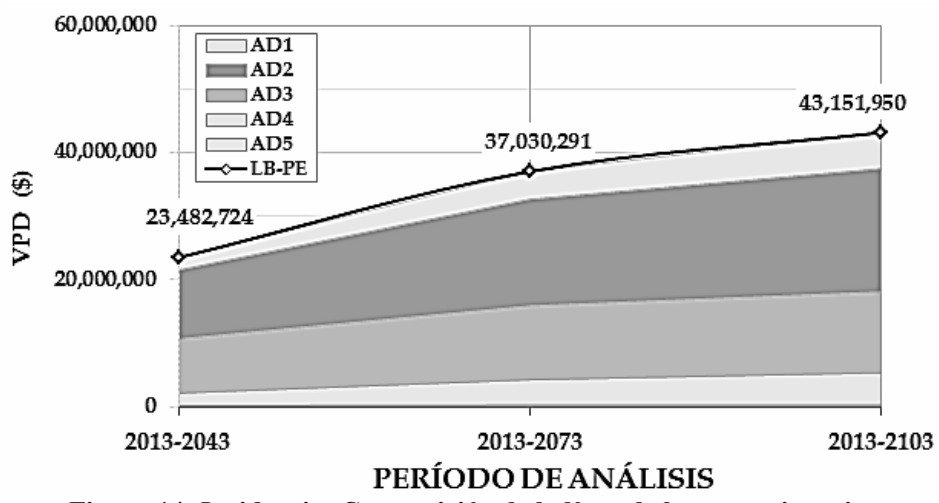

Figura 14: Incidencia: Composición de la línea de base estacionaria.

\section{Valor presente de los daños de las estrategias}

\section{Estrategia E1}

La implementación de la estrategia E1 determinaría los siguientes incrementos porcentuales de las pérdidas en cada horizonte (Figura 15):

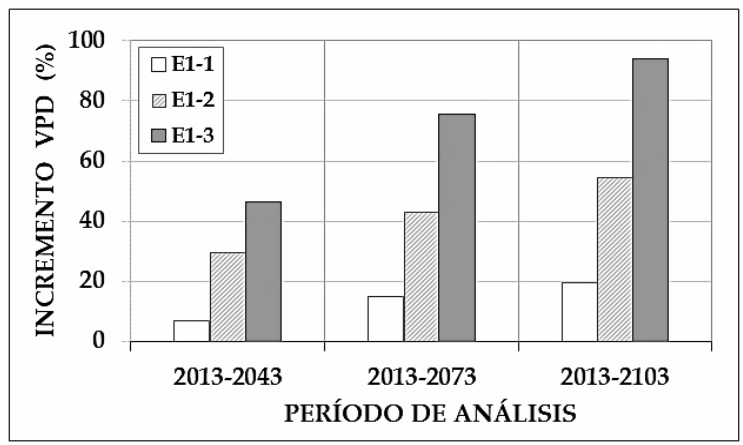

Figura 15: Incremento de los daños acumulados de la estrategia E1 con respecto a la línea de base.

En el corto plazo, los daños a las edificaciones se incrementarían entre un 7\% y 46\%.Las pérdidas acumuladas a mediano plazo crecerían entre el $15 \%$ y el $76 \%$, mientras que a largo plazo se estima que el daño aumentaría entre 55 y $94 \%$.

\section{Estrategia E2}

La implementación de la estrategia E2 reduce el valor presente de los daños en todos los escenarios de cambio climático. La reducción es mayor a medida que se incrementan las dimensiones de las obras, a expensas de un mayor costo de construcción.

Para esta estrategia se estiman las siguientes reducciones porcentuales de los daños acumulados de cada horizonte (Figura 16): en el corto plazo, los daños se reducirían entre $17 \%$ y $69 \%$. A mediano plazo esta reducción estaría comprendida entre el
$16 \%$ y el $58 \%$, mientras que para el largo plazo, las pérdidas disminuirían entre $14 \%$ y $49 \%$.

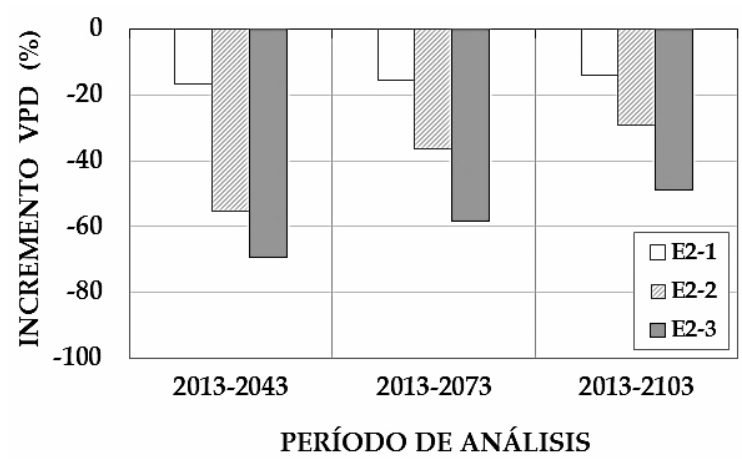

Figura 16: Reducción porcentual de VPD E2 con respecto a la línea de base.

\section{Estrategia E3}

En la Figura 17 se presenta la variación de los daños acumulados con respecto a la línea de base: en el corto plazo, la implementación de esta estrategia implicaría una reducción del los daños de un $17 \%$ para el escenario de cambio climático 1 , e incrementos del $10 \%$ y $7 \%$ para los restantes escenarios.

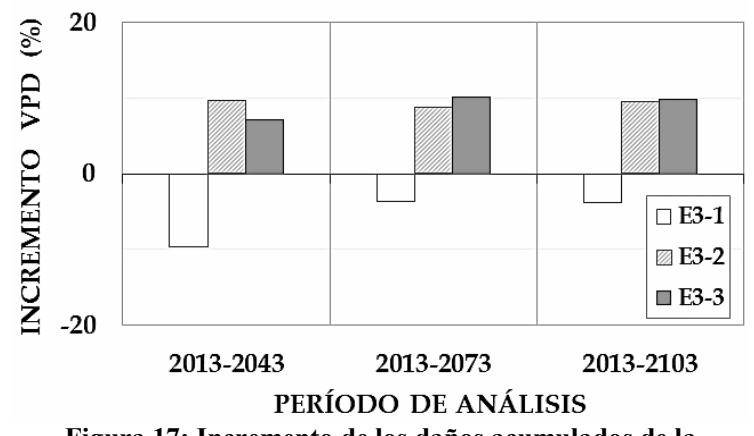

Figura 17: Incremento de los daños acumulados de la estrategia $\mathrm{E} 3$ con respecto a la línea de base. 
A mediano plazo los daños experimentarían una reducción del 4\% para el escenario de cambio climático 1 , e incrementos del $9 \%$ y $10 \%$ para los dos restantes. La variación de los daños a largo plazo con respecto a la línea de base varía entre-4\% $\mathrm{y}+10 \%$.

\section{Estrategia E4}

La variación porcentual de los daños acumulados con respecto a la línea de base se representa en la Figura 18. En el corto plazo, el incremento de los daños es del orden del $2 \%$ en los tres escenarios de cambio climático. A mediano plazo (2013-2073) los daños se incrementarían entre 4\% y $16 \%$. La variación de los daños a largo plazo (2013-2103) con respecto a la línea de base se estima entre $7 \%$ y $29 \%$.

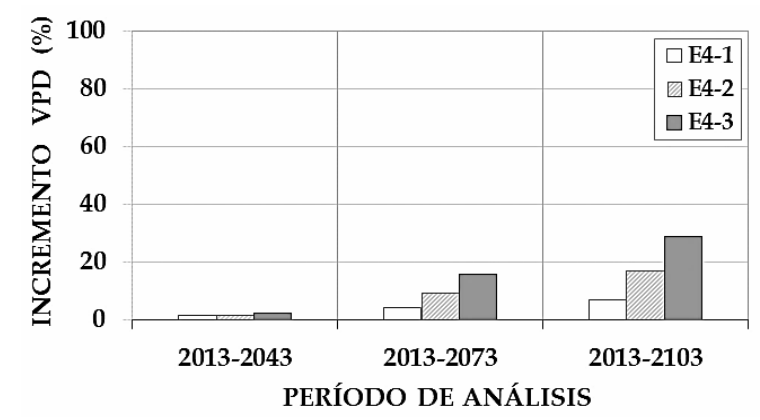

Figura 18: Incremento de los daños acumulados de la estrategia $\mathrm{E} 4$ con respecto a la línea de base.

\section{Costos de implementación de las estrategias}

Como ha sido mencionado, el costo de implementación de cada estrategia ( $\mathrm{C}$ en $\$$ ) se calculó como la diferencia entre el valor presente del costo de construcción (VPC en \$), y el correspondiente a estrategia E1. El costo total de la estrategia E1 asciende a \$96.771.446, y está integrado por:

El costo de las obras de microdrenaje, de $\$ 75.829 .670$ que incluye la rotura y reparación de calles, movimiento de suelos, construcción de cámaras y ejecución de $6778 \mathrm{~m}$ de conductos de sección circular, y de 3764 m de sección rectangular. La longitud total de los colectores de microdrenaje es de 10542 metros, por lo cual el costo medio por cuadra sistematizada es del orden de 689 \$/cuadra.

El costo de construcción del sistema de macrodrenaje, que asciende a \$20.941.776, incluye el movimiento de suelos para la ejecución de los reservorios, construcción de las respectivas obras de descarga y limpieza y perfilado de los tramos del cauce principal.

La Figura 19 representa la variación de los costos de implementación de cada estrategia ( $\mathrm{C}$ en $\$$ ), donde puede observarse su crecimiento en función del escenario de cambio climático.

La estrategia E4 implica los mayores costos de implementación, determinado principalmente por el costo de provisión y colocación de tanques de almacenamiento. En función del escenario de cambio climático, la estrategia E4 incrementa el valor presente del costo de construcción de E1 entre $12 \%$ y $36 \%$.

La estrategia E2 implica un costo de implementación intermedio, cuyo valor total es superior al costo de construcción de E1 entre 4\% y 14\%, en función del escenario de cambio climático.

El menor costo de implementación corresponde a la estrategia $\mathrm{E} 3$, que se incrementa entre $1 \%$ y $11 \%$ con respecto al costo de $\mathrm{E} 1$.

Si bien en el último horizonte temporal del horizonte de planeamiento (2073-2103) las dimensiones de la red corresponden a las de la estrategia E2, las mismas se alcanzan a través de la adaptación y recambio parcial de las obras, en los cortes temporales 2043 y 2073. El descuento de los costos de renovación parcial al año base 2013 reduce el valor presente del costo de construcción de la estrategia E3. 


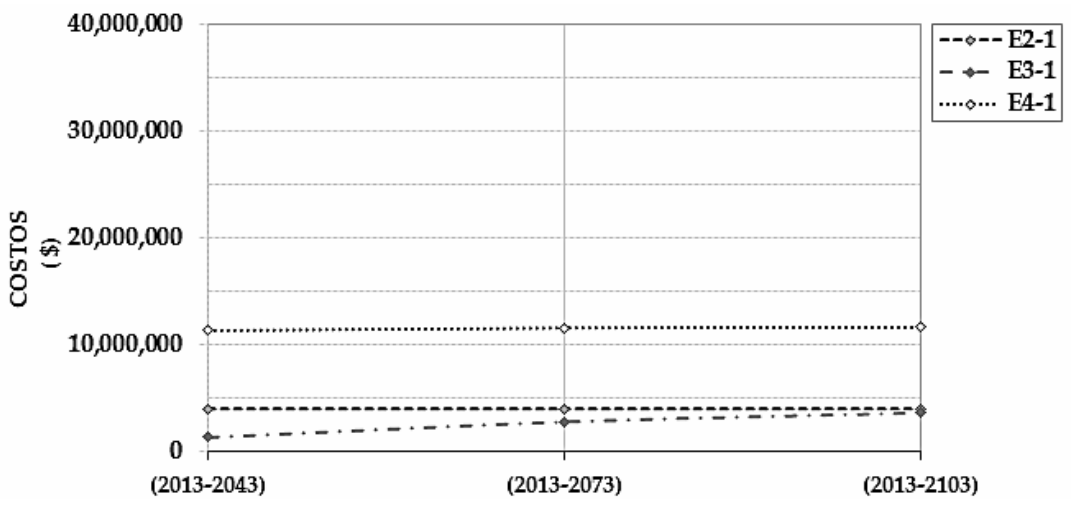

a. Escenario de cambio climático 1.
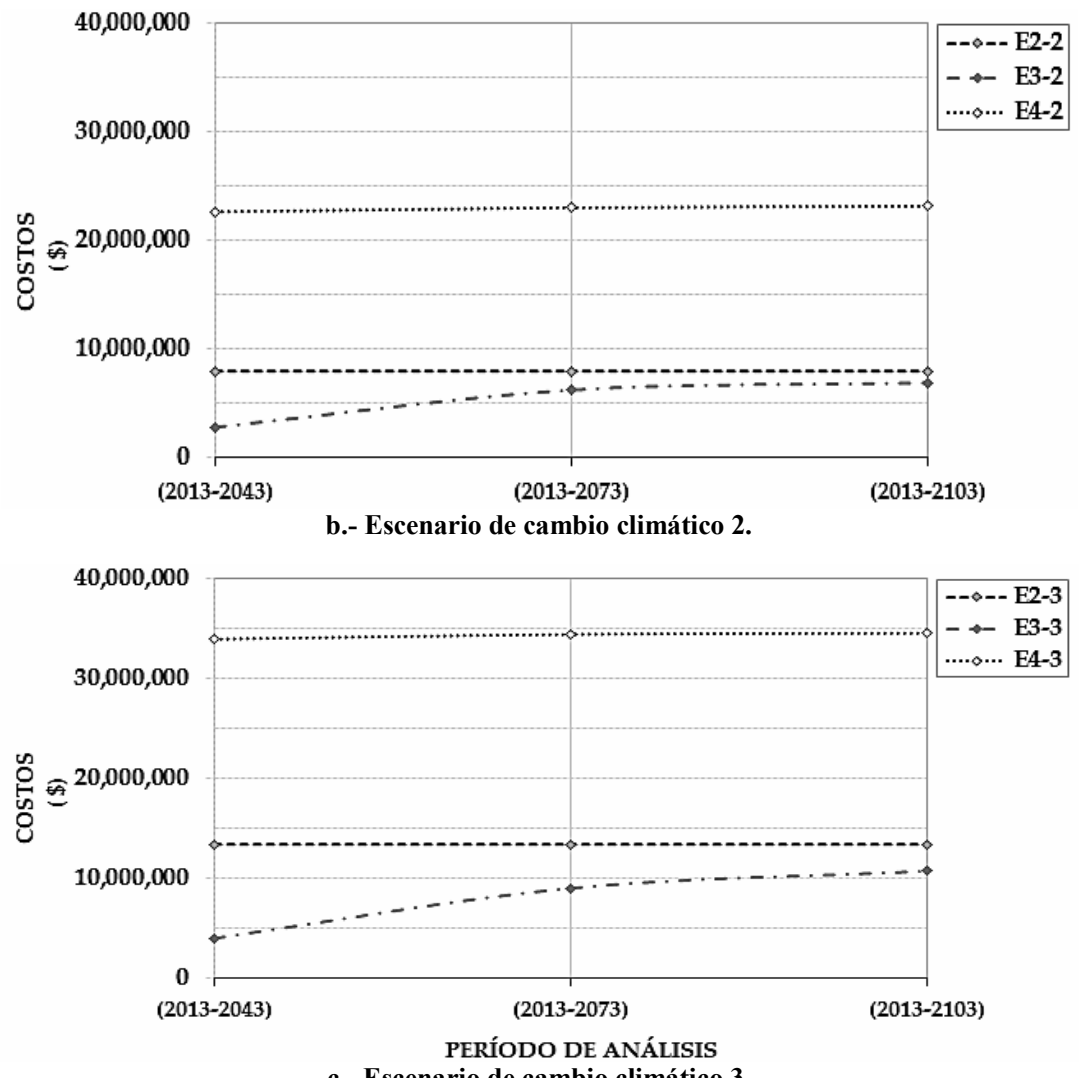

c.- Escenario de cambio climático 3.

Figura 19: Costos de implementación de las estrategias.

Relación costo-beneficio $(\mathrm{B} / \mathrm{C})$ de las estrategias Se utilizó la metodología de análisis costo-beneficio para comparar la relación existente entre los costos y beneficios económicos de cada estrategia en horizontes temporales futuros de los tres escenarios de cambio climático. Para ello se calcularon los cocientes entre los beneficios (costos netos evitados por implementación de cada estrategia con respecto a la situación de no acción, y los respectivos costos de implementación (diferencias de valor presente del costo de construcción con respecto a la situación de no acción).

La variación de las relaciones costo beneficio $(\mathrm{B} / \mathrm{C})$ de las estrategias en cada período del horizonte de planeamiento se presenta en la Figura 20. 


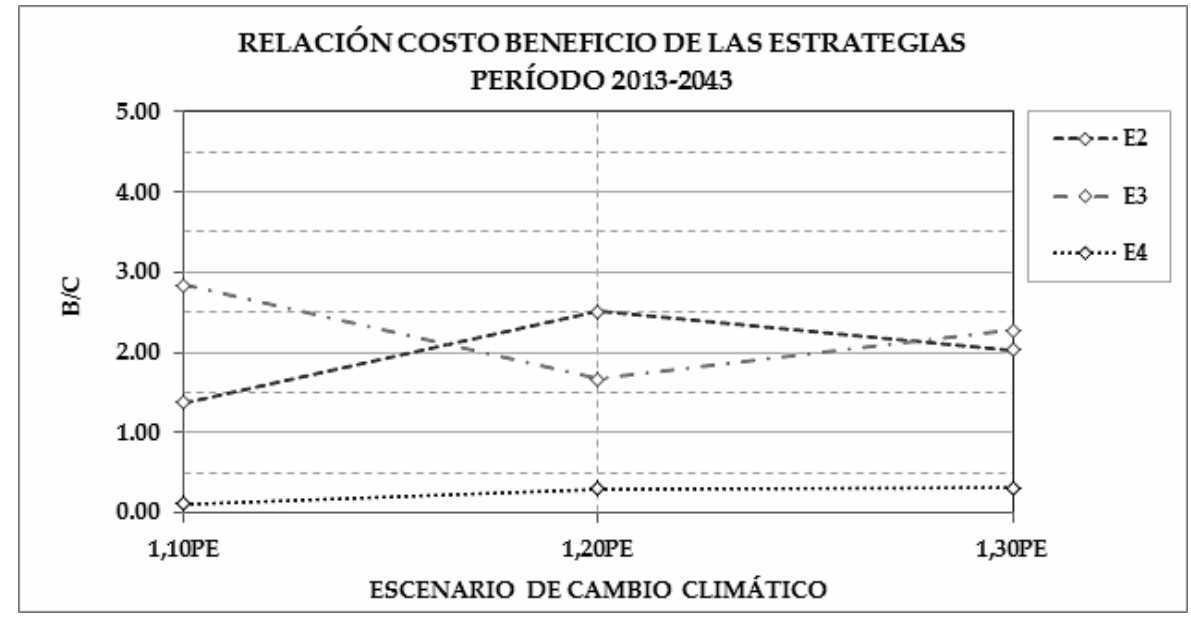

a: Corto plazo 2013-2043.

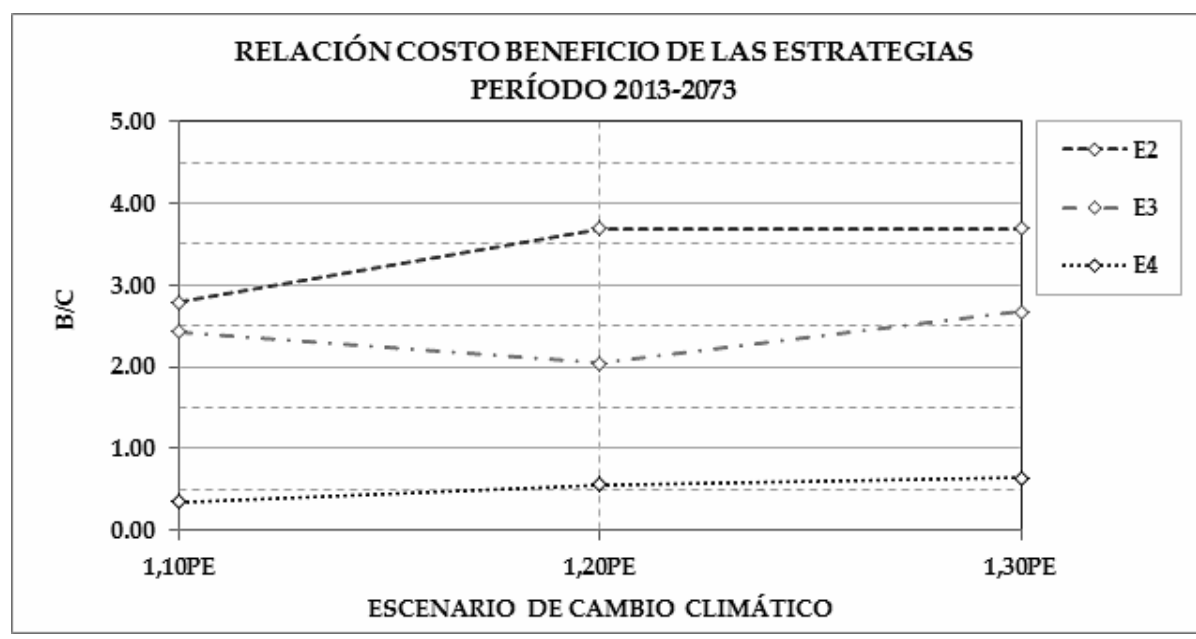

b: Mediano plazo 2013-2073.

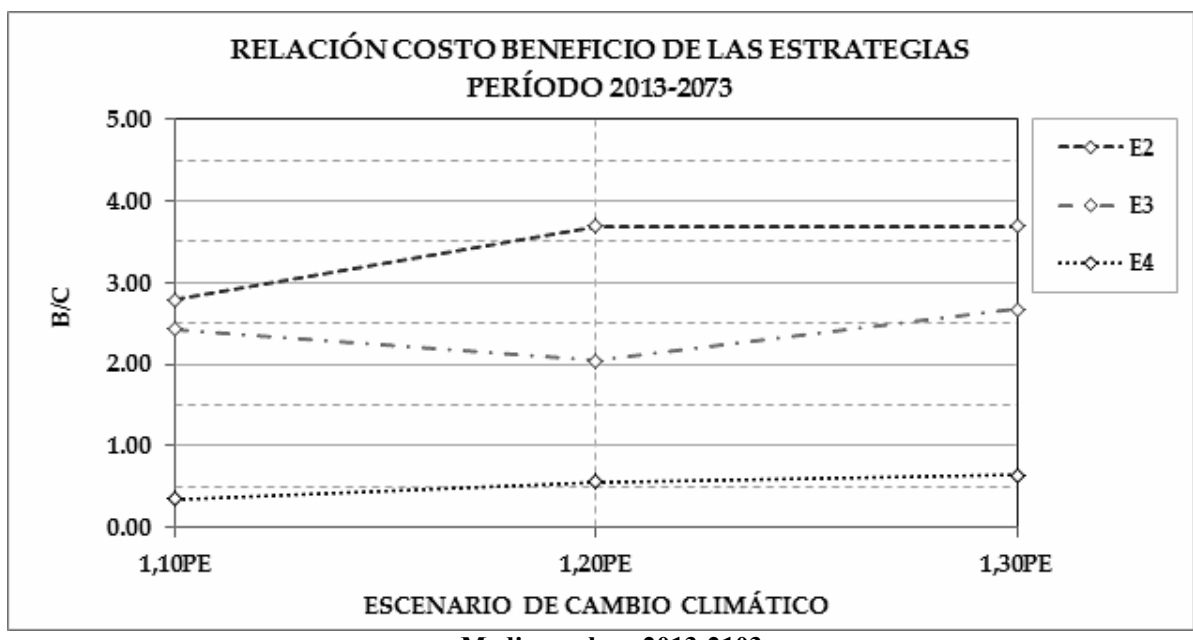

c: Mediano plazo 2013-2103.

Figura 20: Variación de la relación $\mathrm{B} / \mathrm{C}$ con el escenario de cambio climático. 
A partir de la comparación de resultados de este indicador económico es posible presentar una estimación del impacto económico de las tres estrategias de planeamiento en contextos climáticos variables:

La estrategia $\mathrm{E} 4$ presenta relaciones $\mathrm{B} / \mathrm{C}$ inferiores a 1 en todos los escenarios de cambio climático, variables en el rango 0,10-0,80, debido principalmente a que esta estrategia implica los mayores costos de implementación.

A mediano y largo plazo, la estrategia E2 presenta mejores relaciones costo beneficio que la estrategia E3. Mientras las relaciones $\mathrm{B} / \mathrm{C}$ de $\mathrm{E} 2$ varían en el rango 2,8-4,6, las correspondientes a E3 se ubican en el rango $2,4-3,4$

A corto plazo la estrategia E2 presenta mayores relaciones costo beneficio que E3, excepto en el escenario de cambio climático 1 . La reducción general de la relación $\mathrm{B} / \mathrm{C}$ de $\mathrm{E} 2$ en el corto plazo para este escenario se debe a que la estrategia E3 tiene un costo de implementación más bajo como consecuencia de sus menores dimensiones, las cuales se adaptan gradualmente al crecimiento de la precipitación. Es decir, en este horizonte temporal las relaciones $\mathrm{B} / \mathrm{C}$ se ven afectadas por la falta de uniformidad en la respuesta hidráulica de la red de drenaje, determinada principalmente por la capacidad ociosa de los conductos que genera la selección de diámetros comerciales.

\section{Valor presente neto (VPN) de las estrategias}

El valor presente neto (VPN) representa el monto total que el tomador de decisiones estará dispuesto a asumir en caso de adoptar una estrategia en particular: tanto en concepto de inversión en la construcción de la red como en gastos por reparación de los daños. Por lo tanto, las estrategias que presentan un mejor desempeño minimizan el valor presente neto absoluto (VPN en \$).
En la Figura 21 puede observarse que el mayor VPN corresponde a la estrategia $\mathrm{E} 4$, como resultado del costo asociado a la provisión y colocación de tanques de almacenamiento. Su VPN absoluto supera el costo total de la estrategia de no acción, es decir que en caso de adoptar esta estrategia el estado debería gastar un monto total superior en construir la red y reparar los daños por inundación.

La implementación de E2 y E3 reduce el valor presente neto total, por debajo de la estrategia de no acción E1. En el caso de E2, el monto total que el estado debe gastar en inversión en infraestructura y costos de reparación de daños es incluso inferior a los correspondientes a la línea de base estacionaria en los escenarios analizados.

\section{Combinatoria de impactos previstos e impactos reales}

El análisis de indicadores económicos presentado previamente se basa en la hipótesis de que el impacto que causa el cambio es igual al previsto en proyecto. Esta es solo una de las situaciones posibles, casi ciertamente el impacto será diferente al previsto, menor o mayor, en algún porcentaje desconocido (escenario de incerteza profunda).

Si el dimensionamiento de la red se realiza para un impacto previsto específico, y la evolución futura de las precipitaciones resulta en un impacto real de menor magnitud, la consecuencia es un "desperdicio" de dinero por sobredimensionamiento de la red. Sin embargo las obras de mayores dimensiones generan también un "ahorro" de dinero por reducción de los daños.

Si el régimen de precipitaciones intensas evoluciona hacia un impacto real mayor que el adoptado para el dimensionamiento de la red, el costo de los daños se incrementa, sin embargo la inversión en infraestructura es de menor magnitud. 


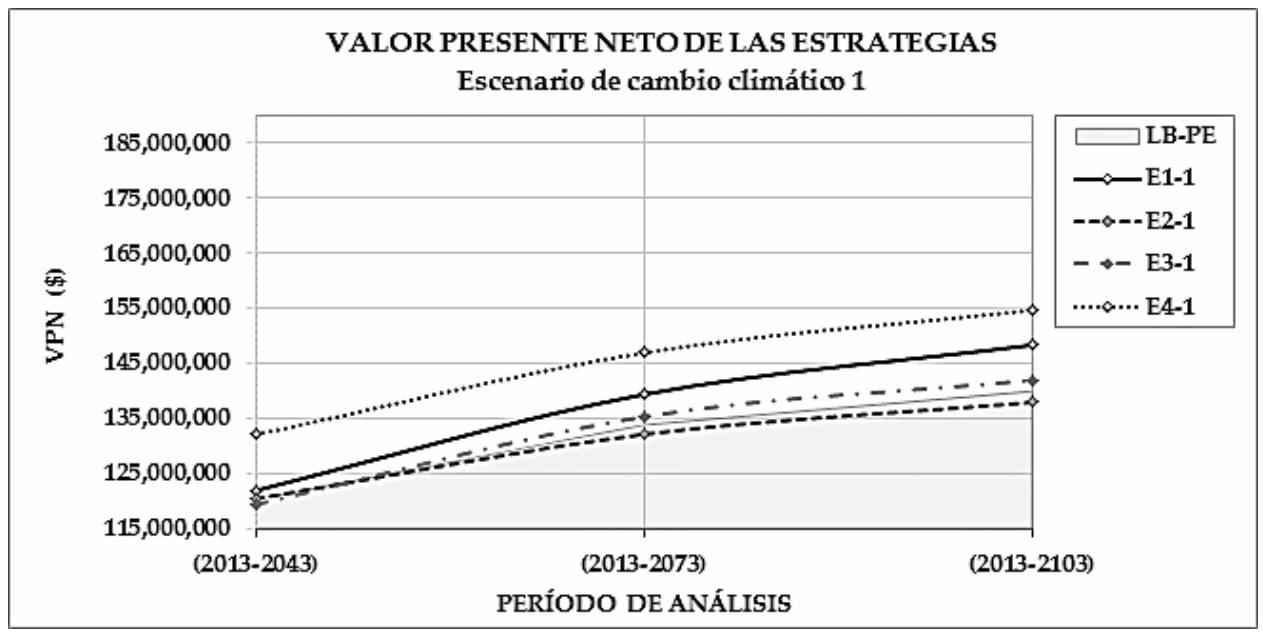

a: Escenario de cambio climático 1.

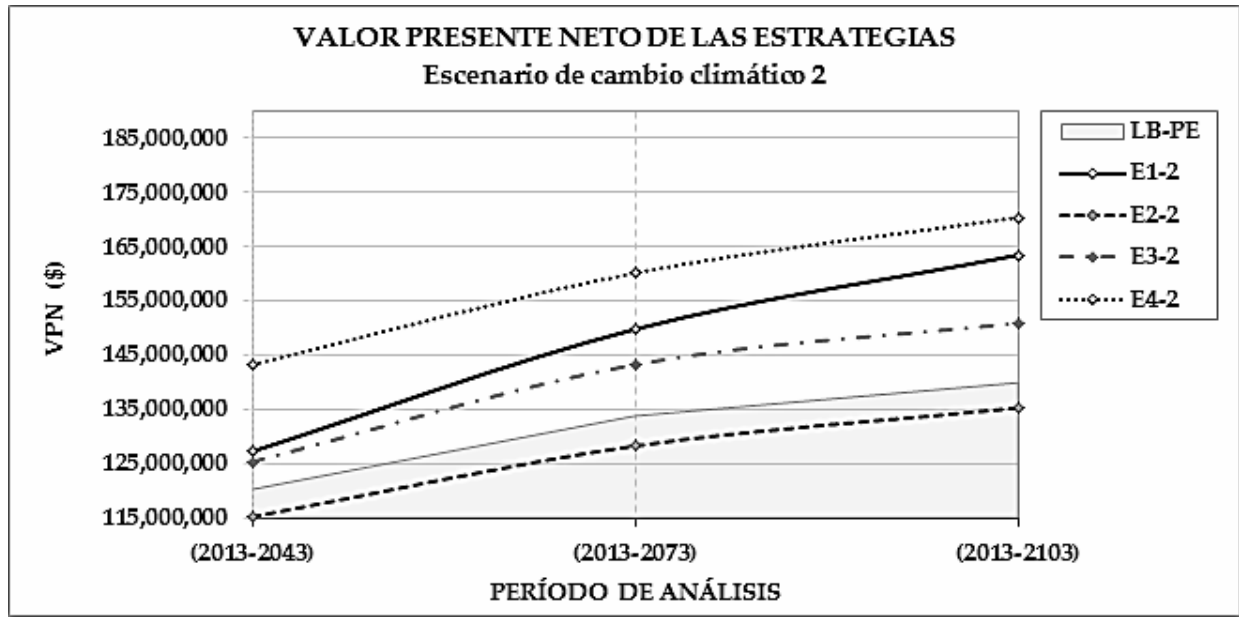

b: Escenario de cambio climático 2.

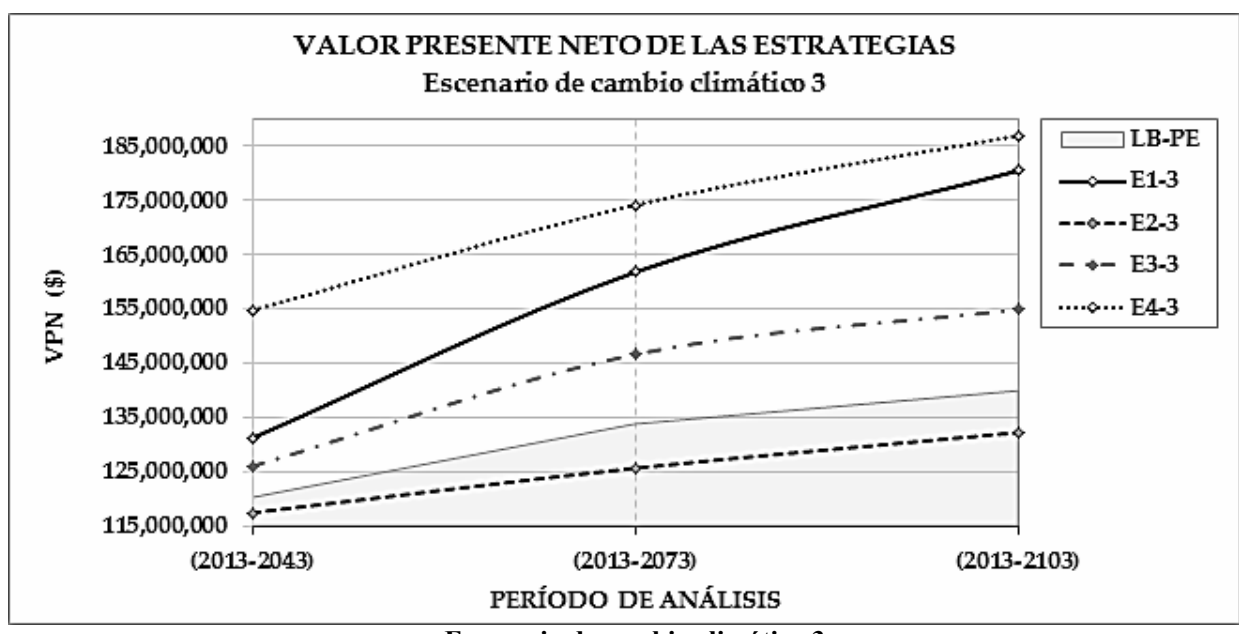

c: Escenario de cambio climático 3.

Figura 21: Valor presente neto de las estrategias. 
Para evaluar las consecuencias económicas de la variación de las precipitaciones futuras sobre las estrategias de diseño, se plantearon las posibles combinaciones entre impactos previstos (en el proyecto de la red) e impactos reales (como consecuencia de la evolución futura de las precipitaciones).

Se simuló el desempeño de cada diseño frente a escenarios futuros diferentes a los asumidos en el proyecto de la red, analizándose la variación de los indicadores económicos (costos, beneficios, $\mathrm{B} / \mathrm{C}, \mathrm{VPN})$.

Los beneficios de cada estrategia se incrementan a medida que aumenta el escenario de cambio climático. Es decir que los casos de impacto previsto mayor al impacto real (redes subdimensionadas) generan beneficios superiores a los correspondientes a las condiciones de proyecto. A la inversa, en el caso de que el impacto real sea menor al previsto (redes sobredimensionadas) se genera una reducción de los beneficios, determinada principalmente por la menor magnitud de los daños de la estrategia de no acción.

El rango de variación de la relación costo beneficio y los correspondientes incrementos porcentuales del VPD con respecto a la estrategia de no acción se resumen en la Figura 22 y Figura 23 respectivamente para el mediano plazo (2013-2073).

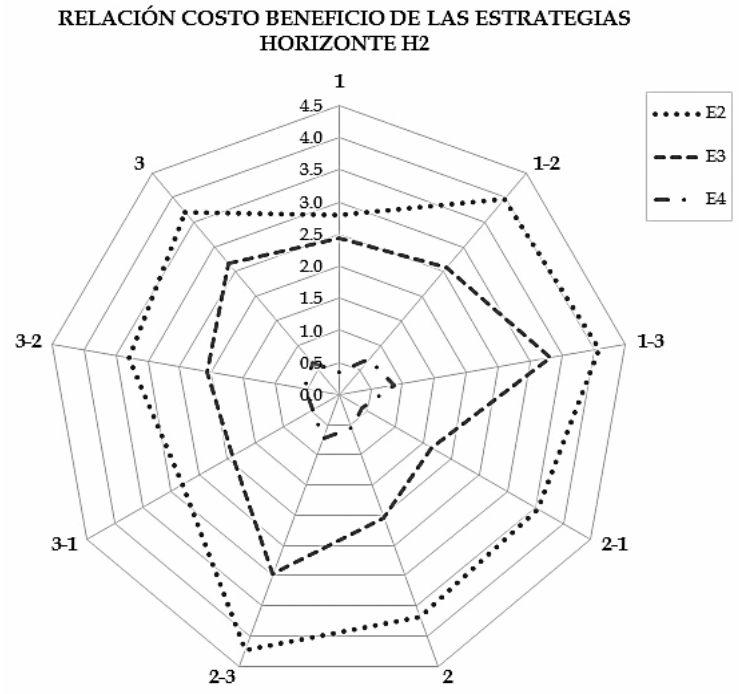

Figura 22: Relación costo beneficio de las estrategias.

En términos generales, los resultados siguen la tendencia de los casos anteriormente analizados: las estrategias E2 y E3 son económicamente más ventajosas, mientras que la E4 tiene una relación $\mathrm{B} / \mathrm{C}$ menor que 1 en todos los casos.

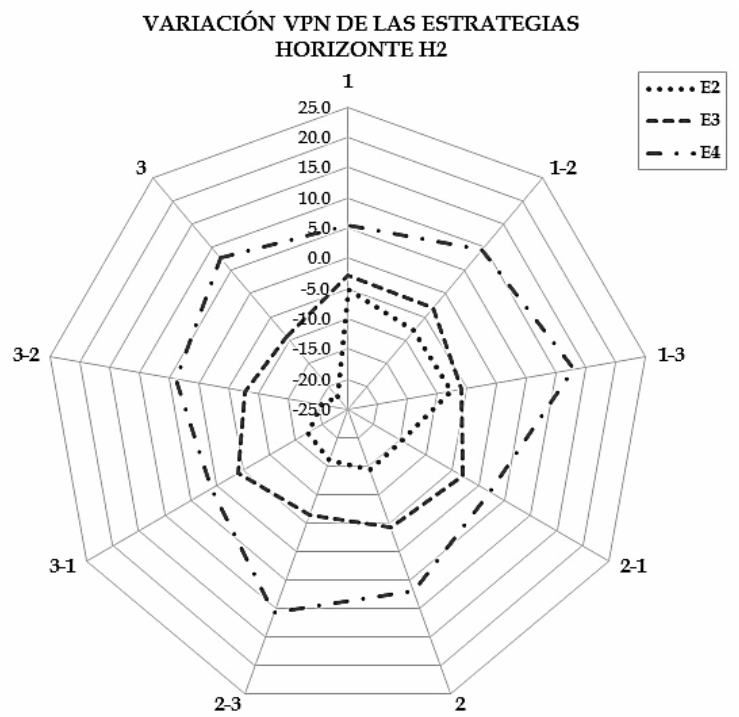

Figura 23: Variación del VPN de las estrategias (\%).

Tanto a corto como a mediano plazo, los beneficios de las estrategias E2 y E3 son superiores a sus costos de implementación. Es decir que el incremento en el costo de construcción de la red es compensado con los beneficios, incluso en el corto plazo. Como consecuencia, todas las combinaciones de ambas estrategias arrojan relaciones $\mathrm{B} / \mathrm{C}$ mayores a 1 .

A su vez, en el caso de las estrategias E2 y E3, todas las combinaciones entre impacto previsto e impacto real presentan una reducción del VPN con respecto a la estrategia de no acción E1. Es decir que si bien se incrementa la inversión necesaria para la construcción de las redes, la reducción de los gastos en reparación de daños determina un costo total menor.

Comparando los resultados de las estrategias E2 y E3, se observa que:

Los beneficios de la estrategia E3 son inferiores a los de la estrategia E2: los mismos equivalen al $40 \%$ y $50 \%$ de los beneficios de la estrategia E2, a corto y mediano plazo respectivamente.

Los costos de implementación de la estrategia E3 son inferiores a los de la estrategia E2: en promedio equivalen a un $33 \%$ del costo de implementación de la estrategia E2 en el corto plazo y a un $72 \%$ en el mediano plazo. 
E2 presenta mejores relaciones $\mathrm{B} / \mathrm{C}$ y menores VPN que la estrategia E3, como consecuencia de la mayor reducción de los daños por inundación.

Si bien para E3 los beneficios son menores, esta estrategia tiene el mínimo costo de implementación y cubre los requisitos de flexibilidad, que permite controlar el incremento de precipitaciones a corto plazo, y puede ser ampliada en el futuro en función de la evolución del cambio climático. Es decir, que aunque los indicadores económicos reflejan un desempeño más bajo, esta estrategia permitiría al tomador de decisiones posponer algunas decisiones de inversión hasta el futuro, cuando es posible que el panorama de impactos sea más claro.

Debido al elevado costo de provisión y colocación de tanques de almacenamiento, los beneficios de la estrategia E4 no son suficientes para compensar sus costos de implementación. Como consecuencia, tanto a corto como mediano plazo, esta estrategia presenta relaciones costo beneficio menores a 1 .

El Valor Presente Neto de todas las combinaciones de la estrategia E4 se incrementa con respecto a la estrategia E1. Es decir que implementar esta estrategia generaría un aumento del costo total con respecto al escenario de no acción, variable entre 5 y $20 \%$ en el corto plazo, y entre $1 \%$ y $13 \%$ en el mediano plazo.

\section{CONCLUSIONES}

En este trabajo se consideraron estrategias de tipo estructural, proponiendo cuatro alternativas específicas de adaptación al cambio climático: estrategia de no acción (E1), estrategia de dimensionamiento para incrementos máximos al final del horizonte de planeamiento (E2), adaptación gradual de la red (E3), y alternativa mixta con incorporación de medidas de control en la fuente $(\mathrm{E} 4)$.

La estrategia de no acción E1 representa un caso extremo, donde el cambio climático es ignorado. Debido a que, en teoría, las dimensiones de la red permanecen constantes durante todo el período de análisis, esta estrategia no tiene flexibilidad para adaptarse a incrementos variables de las precipitaciones. Por lo tanto la magnitud de los daños varía en relación directa con el aumento de las precipitaciones en cada escenario de cambio climático, incrementándose los problemas de anegamiento y daños asociados a medida que aumenta la precipitación.

En el caso de la estrategia E2, como las dimensiones de las obras de drenaje se proyectan para el máximo incremento de precipitación previsto en cada escenario de cambio climático, esta estrategia tiene mayores costos de construcción de la red de drenaje, pero genera la máxima reducción de daños, lo que resulta en una ecuación económica muy favorable.

La implementación de la estrategia E3 implica la adaptación gradual de la red a los incrementos estimados en cada escenario. Esta estrategia también determina una reducción de los daños en todos los horizontes temporales, aunque de menor magnitud que la correspondiente a la estrategia E2. La respuesta del sistema (magnitud del tirante y daño asociado) no varía linealmente con el incremento del escenario de cambio climático. La reducción de los daños se encuentra influenciada por la capacidad ociosa de los conductos, y por las propiedades geométricas e hidráulicas de la calle tipo, secciones del cauce principal y almacenamiento de los reservorios. La ecuación económica es favorable, pero inferior a E2.

La implementación de la estrategia E4 combina los criterios de diseño de las estrategias E1 y E3, dado que la red de microdrenaje se proyecta para la precipitación estacionaria y el macrodrenaje se adapta gradualmente al incremento de las precipitaciones. A su vez incorpora medidas de control en la fuente a escala de cuenca, (tanques de almacenamiento domiciliarios). Esta estrategia presenta beneficios del mismo orden de magnitud que E3 en el caso de escenarios de cambio climático moderado y elevado tanto a corto como a mediano plazo. Sin embargo, la estrategia E4 implica los mayores costos de implementación. Cabe destacar que esta estrategia es altamente dependiente de los dispositivos de control adoptados y de la distribución de los mismos, por lo que otras versiones de esta estrategia podrían generar resultados diferentes.

Con respecto al funcionamiento de las redes en escenarios de impacto real diferente al impacto previsto en el proyecto, el desempeño de las estrategias E2 y E3 es similar: en el caso de las redes sobredimensionadas (impacto real menor al previsto) se genera una reducción de los beneficios, determinada principalmente por la menor magnitud de los daños de la estrategia de no acción. En el caso 
de redes subdimensionadas, que funcionan en escenarios de cambio climático de mayor magnitud a los asumidos en el proyecto de la red (impacto real menor al impacto previsto), se generan beneficios superiores a los correspondientes a las condiciones de proyecto.

En estas combinaciones no se modifica la tendencia general de las estrategias. Comparando los resultados de las estrategias E2 y E3, se observa que aún en los casos de redes sobre y subdimensionadas, E2 presenta menores VPN y mejores relaciones B/C que la estrategia E3, como consecuencia de la mayor reducción de los daños por inundación.

De acuerdo a los resultados de la evaluación económica, la implementación de la estrategia E4 requeriría concentrar los tanques de almacenamiento en las áreas de mayor incidencia en el daño total, dado que su aplicación a escala de cuenca incrementa el costo de construcción sin una reducción significativa de los daños, lo cual reduce el desempeño económico de la estrategia, en particular en los casos de sobredimensionamiento de la red.

En los análisis de largo plazo (H3, 2013-2103), el valor de daños evitados aumenta "vegetativamente" generando cierta distorsión de los resultados. Este aumento a su vez toma como base la rigidez de las estrategias. En este sentido, la estrategia E2 (en particular la E2-3) es la de mayor rigidez, debido a que en el año inicial contempla la construcción de una red para el escenario más desfavorable.

El supuesto de rigidez de las estrategias a lo largo del horizonte de planeamiento 2013-2103 no parece muy realista, dado que siempre es posible modificar la estrategia adoptada; por este motivo, parece más interesante focalizar los análisis en los horizontes $\mathrm{H} 1$ y H2. Por otro lado, no es raro en Argentina que la infraestructura exceda ampliamente su vida útil por falta de renovación; actualmente existen diversos sistemas de desagües, agua potable y cloacas con alrededor de 100 años, claramente obsoletos. Eso da validez al uso del horizonte $\mathrm{H} 3$.

Si bien para E3 los beneficios son menores, esta estrategia tiene el mínimo costo de implementación. Además por tratarse de una estrategia flexible, permite controlar el incremento de precipitaciones a corto plazo, a niveles similares y con un costo menor que E2, pero puede ser ampliada en el futuro en función de la evolución del cambio climático. Es decir, que aunque los indicadores económicos reflejan un desempeño más bajo, esta estrategia permitiría al tomador de decisiones posponer algunas decisiones de inversión hasta el futuro, cuando es posible que el panorama de impactos sea más claro.

A su vez, los costos de implementación de ambas estrategias en los escenarios de cambio climático analizados no superan el $15 \%$ del costo de construcción de la red para la precipitación estacionaria. Ya en el corto plazo ese incremento en el costo es compensado con la disminución de los daños (beneficios) para ambas estrategias (E2 y E3).

Por lo tanto la selección de estrategias en el proceso de toma de decisiones involucrará una serie de aspectos que trascienden el objetivo del análisis costo beneficio clásico. Los criterios de selección dependerán de la aversión al riesgo del tomador de decisiones, tanto en sobreinvertir en protección climática y perder la oportunidad de utilizar los fondos para otros fines, como en la factibilidad de desarrollar los programas de renovación periódica de las redes.

Un aspecto interesante a destacar es que los resultados de este trabajo también son, al menos parcialmente, aplicables a las incertezas generales relacionadas a la lluvia, que van más allá de las originadas por el cambio climático, y ya se encuentran en condiciones de afectar los proyectos. Por ejemplo la utilización de series de lluvias muy cortas y poco representativas, o la variabilidad climática.

Los costos de implementación de E2 y E3 en los escenarios analizados no superan el $15 \%$ del costo de construcción de la red para la precipitación estacionaria. Ya en el corto plazo ese incremento en el costo es compensado con la disminución de los daños (beneficios) para ambas estrategias.

Los análisis comentados aquí han sido hechos considerando que el canal principal de macrodrenaje se mantiene a cielo abierto y en estado semi-natural. El costo de entubamiento sería del orden de $\$ 54$ millones (2013). Un análisis que considerara el macrodrenaje entubado podría dar un resultado diferente en relación a las estrategias de adaptación.

En este trabajo se utilizaron las relaciones adimensionales tirante-daño FLEMOps(+) (Thieken, 2008), que cuantifica el valor de los daños como un porcentaje del valor de las edificaciones. Para la 
aplicación de esta metodología se consideró una distribución uniforme del valor de las propiedades en el área de la cuenca. Análisis futuros más profundos deberían incorporar la variación espacial del costo de las propiedades y desarrollar una mejor adaptación de las curvas tirante-daño a las condiciones locales.

En la metodología propuesta se han considerado solo algunas estrategias de tipo estructural. Las líneas futuras de investigación deberían incorporar las estrategias de planeamiento no estructurales, en particular en lo referente al manejo de las planicies de inundación del macrodrenaje, evitando la excesiva impermeabilización, y la preservación de los cursos de agua en condiciones cuasi naturales deben ser medidas prioritarias siempre que posible.

Otra de las cuestiones que se desprenden del trabajo es la necesidad de preparar adecuadamente las calles como vías de escurrimiento, en particular para tormentas más críticas que la de proyecto. Ese escurrimiento ya ocurre en las circunstancias actuales, y la incerteza adicional introducida por el cambio climático acentúa la necesidad de que esté adecuadamente previsto.

Por otro lado, un análisis robusto, que maximizara no solo las alternativas incluidas en este estudio sino que abarcara todo (o casi) el espectro de alternativas y estrategias sería muy interesante. Eso permitiría tener una visión amplia y extensa del panorama general. Por el volumen de trabajo que eso implica, estaba fuera del alcance de este trabajo. Para un análisis de ese tipo tal vez sería necesario un mayor grado de simplificación en el tratamiento del problema.

Sería conveniente ampliar las métricas y criterios utilizados para evaluar y comparar estrategias, la justificación y consecuencias de cada una.

\section{REFERENCIAS}

Allasia, D., 2002. "Impacto das Incertezas no Custo de uma Rede de Macrodrenagem”. Porto Alegre, Brasil: Universidade Federal do Rio Grande do Sul, Instituto de Pesquisas Hidráulicas.

Arcement, G.J. \& Schneider, V.R., 1989. “Guide for Selecting Manning's Roughness Coefficients for Natural Channels and Flood Plains". U.S. GEOLOGICAL SURVEY WATER-SUPPLY, PAPER 2339.
Barros, V.R.; Boninsegna, J.A.; Camilloni, I.A.; Chidiak, M.; Magrín, G.O.; Rusticucci, M., 2014. "Climate change in Argentina: trends, projections, impacts and adaptation". WIREs Clim Change 2014. doi: 10.1002/wcc.316. John Wiley \& Sons, Ltd.

CEPAL, N.U., 2014. "La economía del cambio climático en la Argentina. Primera aproximación". Santiago de Chile: Naciones Unidas.

CIMA, 2015. "Cambio climático en Argentina; tendencias y proyecciones" Tercera Comunicación Nacional Sobre Cambio Climático. Buenos Aires, Argentina. Centro de Investigaciones del Mar y la Atmósfera, Secretaría de Ambiente y Desarrollo Sustentable de la Nación.

Garat, M.E., 2017. "Incorporación de la incerteza del cambio climático en la gestión del drenaje urbano". Rosario, Argentina: Tesis de doctorado, FCEIA, Universidad Nacional de Rosario.

Hallegatte, S., 2009. "Strategies to adapt to an uncertain climate change". Global Environmental Change 19.

Olsen, A.S., 2014. "Comparing Methods of Calculating Expected Annual Damage". Technical University of Denmark.

Thieken, A.H., 2008. "Floods, flood losses and flood risk management in Germany". Potsdam, Alemania: University of Potsdam.

Thieken, A.H.; Kreibich, H.; Müller, M.; Nicklisch, M.; Merz, B., 2007. "Flood losses in private households: analysis of influencing factors and implications for flood loss modelling". Bochum, Germany: International Association of Hydrological Sciences IAHS, Publ. 317.

UDFCD, 2008. URBAN DRAINAGE AND FLOOD CONTROL DISTRICT "Urban Storm Drainage. Criteria Manual". Denver Colorado, USA.

Waters, D.,Watt, W., Marsalek, J, Anderson, B., 2003. "Adaptation of a Storm Drainage System to Accommodate Increased Rainfall Resulting from Climate Change". New York, USA: Journal of Environmental Planning and Management 46 (5): 755-770. Routledge, Taylor \& Francis Group LLC.

Zamanillo, E.; Larenze, G.; Tito, M.J,; Perez, M.; Garat, M.E., 2008. "Tormentas de diseño para la Provincia de Entre Ríos". Concordia, Argentina: Universidad Tecnológica Nacional.

Artículo recibido el 05/2018 y aprobado para su publicación el 07/2018. 